ARTICLE

\title{
Structure of the respiratory MBS complex reveals iron-sulfur cluster catalyzed sulfane sulfur reduction in ancient life
}

\author{
Hongjun Yu,3,4, Dominik K. Haja ${ }^{2,4}$, Gerrit J. Schut ${ }^{2}$, Chang-Hao Wu², Xing Meng ${ }^{1}$, Gongpu Zhao (D) ${ }^{1}$, \\ Huilin Li (i) ${ }^{1 \otimes} \&$ Michael W. W. Adams (1D) ${ }^{2 \otimes}$
}

Modern day aerobic respiration in mitochondria involving complex I converts redox energy into chemical energy and likely evolved from a simple anaerobic system now represented by hydrogen gas-evolving hydrogenase $(\mathrm{MBH})$ where protons are the terminal electron acceptor. Here we present the cryo-EM structure of an early ancestor in the evolution of complex I, the elemental sulfur $\left(\mathrm{S}^{0}\right)$-reducing reductase MBS. Three highly conserved protein loops linking cytoplasmic and membrane domains enable scalable energy conversion in all three complexes. MBS contains two proton pumps compared to one in $\mathrm{MBH}$ and likely conserves twice the energy. The structure also reveals evolutionary adaptations of $\mathrm{MBH}$ that enabled $S^{0}$ reduction by MBS catalyzed by a site-differentiated iron-sulfur cluster without participation of protons or amino acid residues. This is the simplest mechanism proposed for reduction of inorganic or organic disulfides. It is of fundamental significance in the iron and sulfur-rich volcanic environments of early earth and possibly the origin of life. MBS provides a new perspective on the evolution of modern-day respiratory complexes and of catalysis by biological iron-sulfur clusters.

\footnotetext{
${ }^{1}$ Structural Biology Program, Van Andel Institute, Grand Rapids, MI, USA. ${ }^{2}$ Department of Biochemistry and Molecular Biology, University of Georgia, Athens, GA, USA. ${ }^{3}$ Present address: Department of Biochemistry and Molecular Biology, School of Basic Medicine and Tongji Medical College, Huazhong University of Science and Technology, Wuhan, China. ${ }^{4}$ These authors contributed equally: Hongjun Yu, Dominik K. Haja. ${ }^{凶}$ email: Huilin.Li@vai.org; adamsm@uga.edu
} 
espiratory complexes couple the energy released from spontaneous electron transfer reactions to the generation of chemical gradients. From archaea and bacteria to mammalian mitochondria, modern-day respiratory complex I contains a 14-subunit core ${ }^{1-6}$ that oxidizes $\mathrm{NADH}$, reduces quinone, and translocates four protons across the membrane, used to drive ATP synthesis thereby conserving energy ${ }^{7-11}$. Such complexes first appeared billions of years ago in an anaerobic environment where, without oxygen, oxidative microbial metabolism ultimately reduced the ubiquitous proton to produce hydrogen gas catalyzed by a membrane-bound [NiFe]-hydrogenase (Fig. 1a, $\mathrm{MBH})^{6,12-15}$. As the atmosphere became more oxidized, ancestral $\mathrm{MBH}$ evolved to reduce elemental sulfur $\left(\mathrm{S}^{0}\right)$, now represented by sulfane sulfur reductase or MBS, and ultimately to reducing quinone, as in complex $\mathrm{I}^{12-15}$ and related $\mathrm{NDH}^{16}$.
$\mathrm{MBH}$ and MBS are found in the anaerobic hyperthermophilic archaeon Pyrococcus furiosus ${ }^{17}$, which oxidizes the redox protein ferredoxin, produces $\mathrm{H}_{2} \mathrm{~S}$ or $\mathrm{H}_{2}$, and conserves energy by pumping $\mathrm{Na}^{+}$(Fig. 1a) ${ }^{9,12,18,19}$. In the presence of $\mathrm{S}^{0}$, the genes encoding $\mathrm{MBS}$ are expressed while those encoding $\mathrm{MBH}$ are repressed ${ }^{19-21}$, resulting in a microbial cell yield twice that when cells are grown without $S^{0} 9,22$, suggesting that MBS is more efficient in energy conservation although the underlying mechanism is unknown. MBS reductively cleaves linear polysulfide chains $\left(\mathrm{S}_{\mathrm{n}}{ }^{2-}\right.$, where $\left.n \geq 4\right)$, produced abiotically from the reaction of $\mathrm{H}_{2} \mathrm{~S}$ and $\mathrm{S}^{0}$ (Eq. 1) in the hydrothermal vent environments that $P$. furiosus inhabits, into two shorter polysulfides (Eq. 2). Further reduction yields unstable di- and trisulfides (Eq. 3) that spontaneously convert to $\mathrm{S}^{0}$ and $\mathrm{H}_{2} \mathrm{~S}$ (Eq. 4). S-S bond cleavage by MBS does not involve protons (Eqs. 2 and 3)
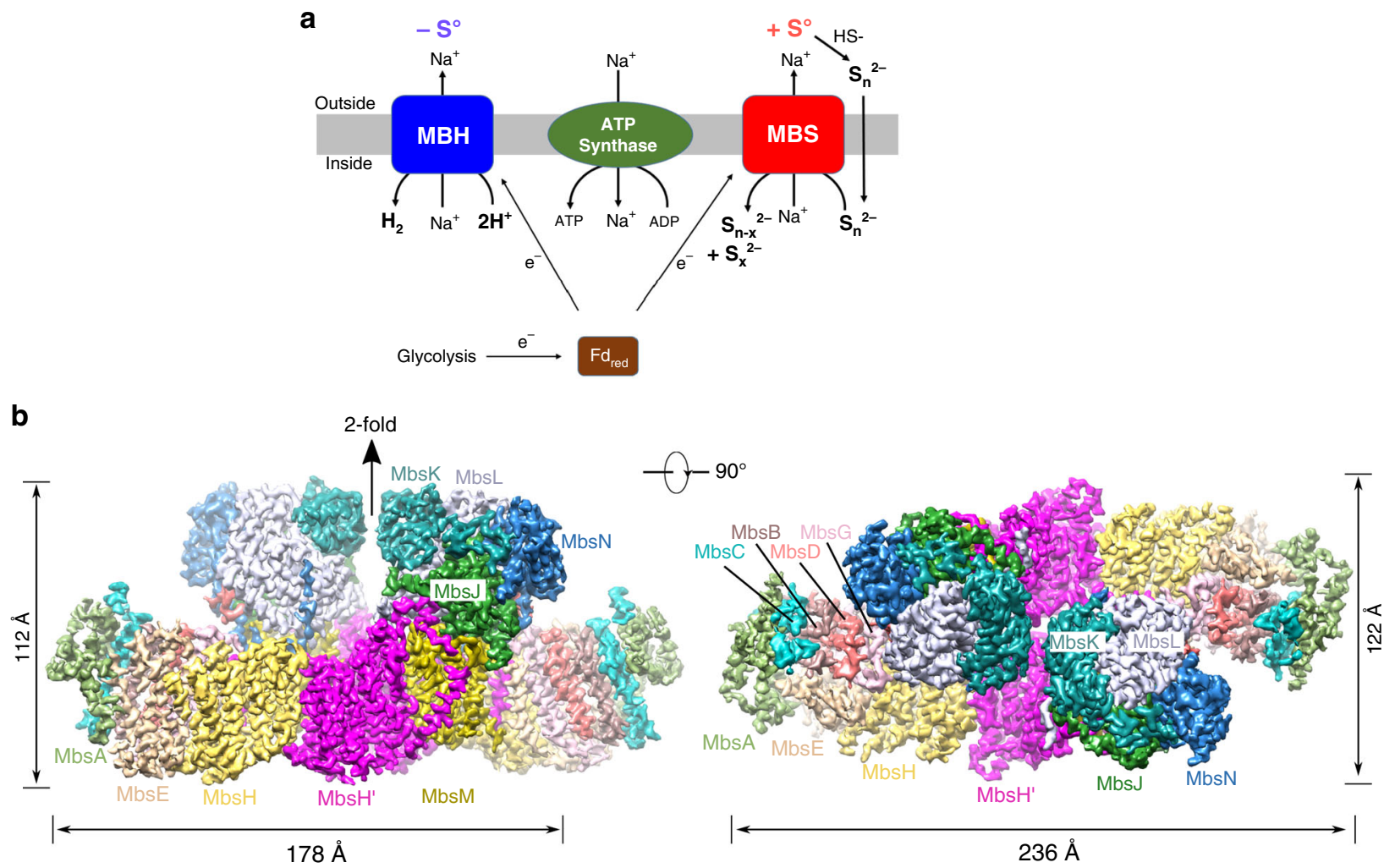

C

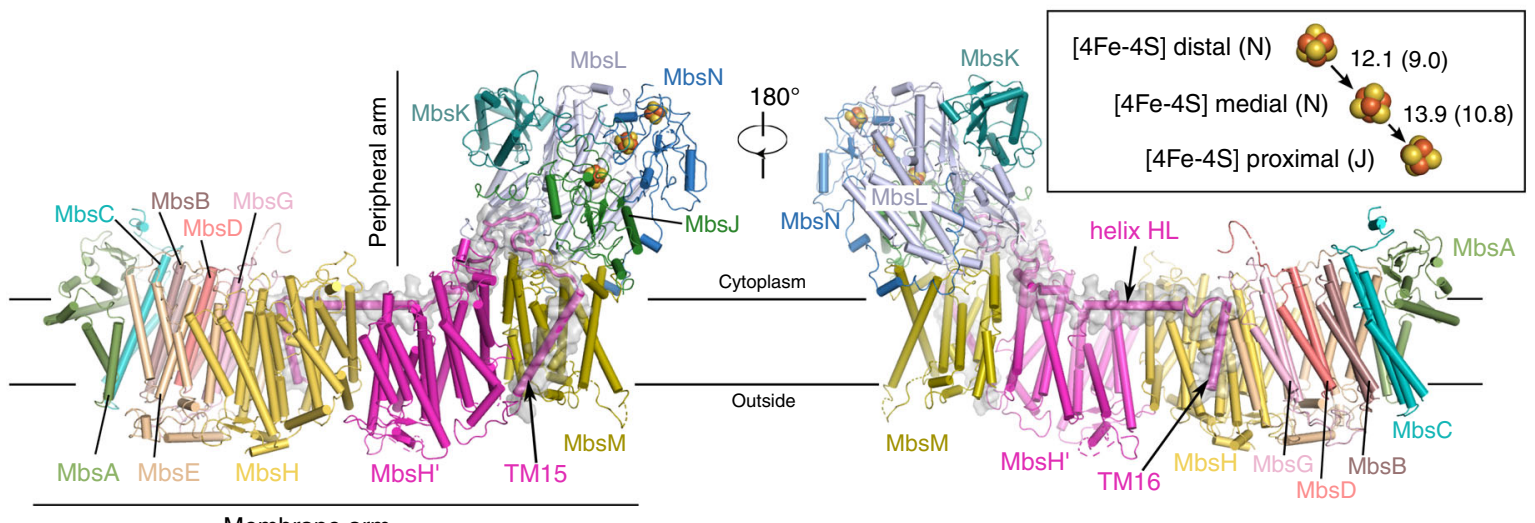

Fig. 1 Overall structure of the $\boldsymbol{P}$. furiosus MBS complex. a Schematic comparison of $P$. furiosus $M B H$ and $M B S$ complexes, both generating a Na ${ }^{+}$gradient for ATP synthesis. b Side (left) and top (right) views of the cryo-EM map of the dimeric MBS complex, segmented and colored by subunits. c The front (left) and back (right) view of the atomic model of an MBS monomeric complex. Individual subunits are colored as in (b). Inset in the right panel: a chain of three $[4 \mathrm{Fe}-4 \mathrm{~S}]$ clusters in the peripheral arm, with the center-to-center and edge-to-edge distances (in brackets) between metal sites indicated in $\AA$. 
and $\mathrm{H}_{2} \mathrm{~S}$ is produced abiotically (Eq. 4). Bacterial polysulfide reductase (PSR, not present in P. furiosus) uses a molybdopterin cofactor to reductively cleave the terminal sulfur of polysulfides as $\mathrm{H}_{2} \mathrm{~S}$ (Eq. 5) ${ }^{23,24}$ so the archaeal MBS must reduce a disulfide bond by a different mechanism.

$$
\text { Abiotic : } \mathrm{S}_{8}+\mathrm{H}_{2} \mathrm{~S} \rightarrow^{-} \mathrm{S}-\mathrm{S}-\mathrm{S}-\mathrm{S}-\mathrm{S}-\mathrm{S}-\mathrm{S}-\mathrm{S}-\mathrm{S}^{-}\left(\mathrm{S}_{9}^{2-}\right)+2 \mathrm{H}^{+}
$$

$$
\begin{gathered}
\text { MBS : } \mathrm{S}_{9}^{2-}+2 e^{-} \rightarrow^{-} \mathrm{S}-\mathrm{S}-\mathrm{S}-\mathrm{S}^{-}+{ }^{-} \mathrm{S}-\mathrm{S}-\mathrm{S}-\mathrm{S}-\mathrm{S}^{-}\left(\mathrm{S}_{5}^{2-}\right) \\
\text { MBS }: \mathrm{S}_{5}^{2-}+2 e^{-} \rightarrow^{-} \mathrm{S}-\mathrm{S}-\mathrm{S}^{-}+{ }^{-} \mathrm{S}-\mathrm{S}^{-}\left(\mathrm{S}_{2}^{2-}\right) \\
\text { Abiotic }: 5 \mathrm{~S}_{2}^{2-}+4 \mathrm{H}^{+} \rightarrow \mathrm{S}_{8}+2 \mathrm{H}_{2} \mathrm{~S} \\
\text { PSR : } \mathrm{S}_{\mathrm{n}}^{2-}+2 \mathrm{e}^{-}+2 \mathrm{H}^{+} \rightarrow \mathrm{S}_{\mathrm{n}-1}^{2-}+\mathrm{H}_{2} \mathrm{~S}
\end{gathered}
$$

The structure of $\mathrm{MBH}$ (298 kDa, 14 subunits) revealed its close evolutionary relationship with complex I. Although $\mathrm{MBH}$ contains a single proton pump compared to four in complex I, they have the same structural elements thought to enable transduction of redox energy to an ion gradient ${ }^{6,7,25-27}$. Herein we describe the structure of MBS ( $357 \mathrm{kDa}, 13$ subunits), the proposed evolutionary link between them ${ }^{9}$, and how a catalytic site evolved from reducing protons to reducing large hydrophobic molecules like $\mathrm{S}_{\mathrm{n}}{ }^{2-}$ and cleaving an inorganic sulfur-sulfur bond, a reaction of high significance in the primordial early earth.

\section{Results}

Structure of MBS. We determined the cryo-EM structure of MBS in a dimer complex form (Fig. 1b, c, Supplementary Fig. 1, Supplementary Fig. 2, Supplementary Table 1). The atomic model contained 26 protein subunits and 116 transmembrane helices (TMHs). The MBS monomer consists of a peripheral cytoplasmic $\operatorname{arm}(\mathrm{MbsJ}, \mathrm{K}, \mathrm{L}$, and $\mathrm{N}$ ), anchored to the membrane by MbsM, and coordinates three $[4 \mathrm{Fe}-4 \mathrm{~S}]$ clusters that transfer electrons from ferredoxin to the substrate polysulfide (Fig. 1c) ${ }^{19}$. The membrane arm (MbsA, B, C, D, E, G, H, H' and M) has 58 TMHs and contains a module proximal to the peripheral arm (proximal membrane module; MbsH', MbsH, MbsD, MbsG and TMH1-2 of $\mathrm{MbsE}$ ) and a distal membrane module (MbsA, B and C, and TMH3-6 of MbsE) (Fig. 2a, Supplementary Fig. 3a).

The MBS dimer complex is assembled via extensive contacts at two main places between the proximal membrane module of one MBS and the membrane-anchored peripheral module of the other (Figs. 1b, 2a-c). The first contact involves a long amphipathic horizontal helix (termed helix HL) of MbsH' (Fig. 2d): the MbsH' helix HL of one MBS interacts extensively with the N-terminal region of $\mathrm{MbsN}$ and TMH8 of MbsM in the other MBS, including several hydrophobic interactions and a salt bridge. The twofold axis is located between two F566 residues preceding the helix HL in the two MbsH' of the MBS dimer complex. The second contact involves the tryptophan-rich C-terminal extension (W90, W91, and W94) of MbsD of one MBS projecting into a largely hydrophobic cleft in the peripheral domain of the other (Fig. 2e). The distal membrane module is not involved in the MBS dimer interface and is likely somewhat dynamic, which may explain the lower local resolution in this region (Supplementary Fig. 1g).

MbsH and MbsH' each contain a proton pump. In the proximal membrane module, the two largest subunits $\mathrm{MbsH}$ and $\mathrm{MbsH}^{\prime}$ are of similar fold, consistent with the idea from sequence comparison that $\mathrm{MbsH}$ is a gene-duplication product of $\mathrm{MbsH}^{19}$ (Supplementary Fig. 3b) that occurred prior to the evolution of $\mathrm{MBH}$ and MBS and, as discussed below, during the evolution of the $\mathrm{Na}^{+} / \mathrm{H}^{+}$Mrp antiporter. $\mathrm{MbsH}$ and $\mathrm{MbsH}$ ' are homologous to the antiporter-like subunits of complex I (T. thermophilus $\mathrm{Nqo12}, 13$ and 14) proposed for $\mathrm{H}^{+}$pumping ${ }^{1-3,5}$. MbsH and MbsH' each contain two five-TMH repeating units: TMHs 4-8 and TMHs 9-13 that are superimposable when one repeat is flipped upside down in the membrane (Supplementary Fig. 3b). Each 5-TMH unit features a discontinuous a-helix-TMH7 in the first repeat and TMH12 in the second repeat with conserved charged residues around the helical kink: E219 and K393 in $\mathrm{MbsH}$, and K217 and K399 in MbsH'. Notably, the arrangement between $\mathrm{MbsH}$ and $\mathrm{MbsH}$ ' as a unit is strikingly similar to Nqo13 and Nqo14 of T. thermophilus complex I (Fig. 3a, b). As a result, a central charged axis across $\mathrm{MbsH}$ and $\mathrm{MbsH}^{\prime}$ (MbsH E138, K250, and $\mathrm{H} 330$; MbsH' E128, K248, and H336) is formed that is conserved in complex I (Fig. 3c). These features are characteristic of the proton pumps that are found in mammalian and bacterial forms of complex I, which have been supported by structural studies, mutagenesis analysis, and recent molecular dynamics simulations ${ }^{1-3,28-30}$. We therefore propose that $\mathrm{MbsH}$ and MbsH' each contains a $\mathrm{H}^{+}$pump in MBS.

Interestingly, MbsH' contains two extra TMHs (TMH15 and TMH16) that are connected by a long intervening loop

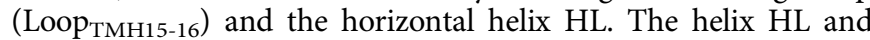
its following TMH16 holds $\mathrm{MbsH}$ and $\mathrm{MbsH}$ ' together. This element is conserved across the different respiratory complexes, with the helix HL either shortened in $\mathrm{MBH}$ (with one antiporterlike subunit $\mathrm{MbhH}$ ) or elongated in complex I (with three antiporter-like subunits) (Fig. 3a, b, Supplementary Fig. 4a), strongly supporting the modular evolution of these respiratory


terminal end of MbsH' tie together the membrane-anchored peripheral module and the proton-pumping module (Supplementary Fig. 4a). This organization mode is conserved in the $\mathrm{MBH}$ complex, though MbsM and MbsH' form a tighter interacting interface than the lipid-filled wide cleft between $\mathrm{MbhM}$ and $\mathrm{MbhH}$ in the $\mathrm{MBH}$ complex (Supplementary Fig. $4 \mathrm{~b})^{6}$. In contrast, in complex I, the membrane-anchored peripheral module is located on the opposite side of the membrane arm (Fig. 3a). However, in spite of this different architecture, a charged axis within the membrane-anchored peripheral arm (MbsM E203, E211, D196, E193, and E104) extends continuously into the proton-pumping module ( $\mathrm{MbsH}$ and $\mathrm{MbsH}$ ) (Fig. 3c), similar to that proposed in complex I for energy transduction ${ }^{1-3,32}$.

The tentative $\mathrm{Na}^{+} / \mathrm{H}^{+}$antiporter module. Except for $\mathrm{MbsH}$ and $\mathrm{MbsH}$, the remainder of the proximal membrane module of MBS is composed of two layers of helix bundles next to $\mathrm{MbsH}$ : one layer composed of three $\mathrm{TMHs}$ of $\mathrm{MbsD}$ and the other layer composed of five TMHs-TMH1-2 of MbsE separated by TMH13 of $\mathrm{MbsG}$ in the middle (Supplementary Fig. 3a). This subregion together with $\mathrm{MbsH}$ is conserved among $\mathrm{MBH}$ and complex I including key elements: charged residues E58 in MbsD, E37 in MbsG, and a $\pi$-bulge distortion in TMH3 of MbsD (Supplementary Fig. 4a and c). These are key features proposed for proton transfer that are conserved in Nqo10 and 11 in complex I and also in $\mathrm{MbhD}, \mathrm{G}$ and $\mathrm{E}$ in $\mathrm{MBH}^{2,3,6,33}$, although the precise mechanism is still under debate ${ }^{1,2,33}$. This suggests a putative proton translocation path, termed Pc path for composite proton translocator. We note that the assignment of the $\mathrm{Pc}$ proton path is based on structural conservation among $\mathrm{MBH}$, MBS, and complex I, and therefore, this path is tentative. The translocation stoichiometry of the $\mathrm{H}^{+}$and $\mathrm{Na}^{+}$is currently unknown and has yet to be experimentally determined with either Mrp, MBH, or MBS. The stoichiometry will help to determine if $\mathrm{Pc}$ is a functional proton path. 

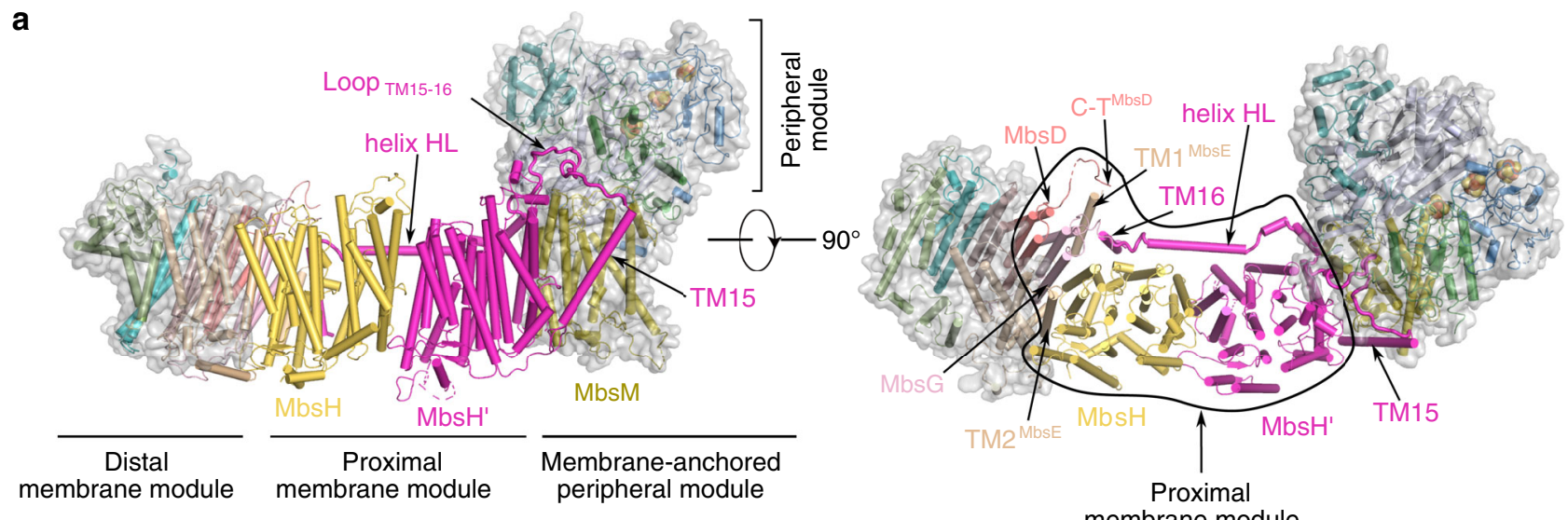

b
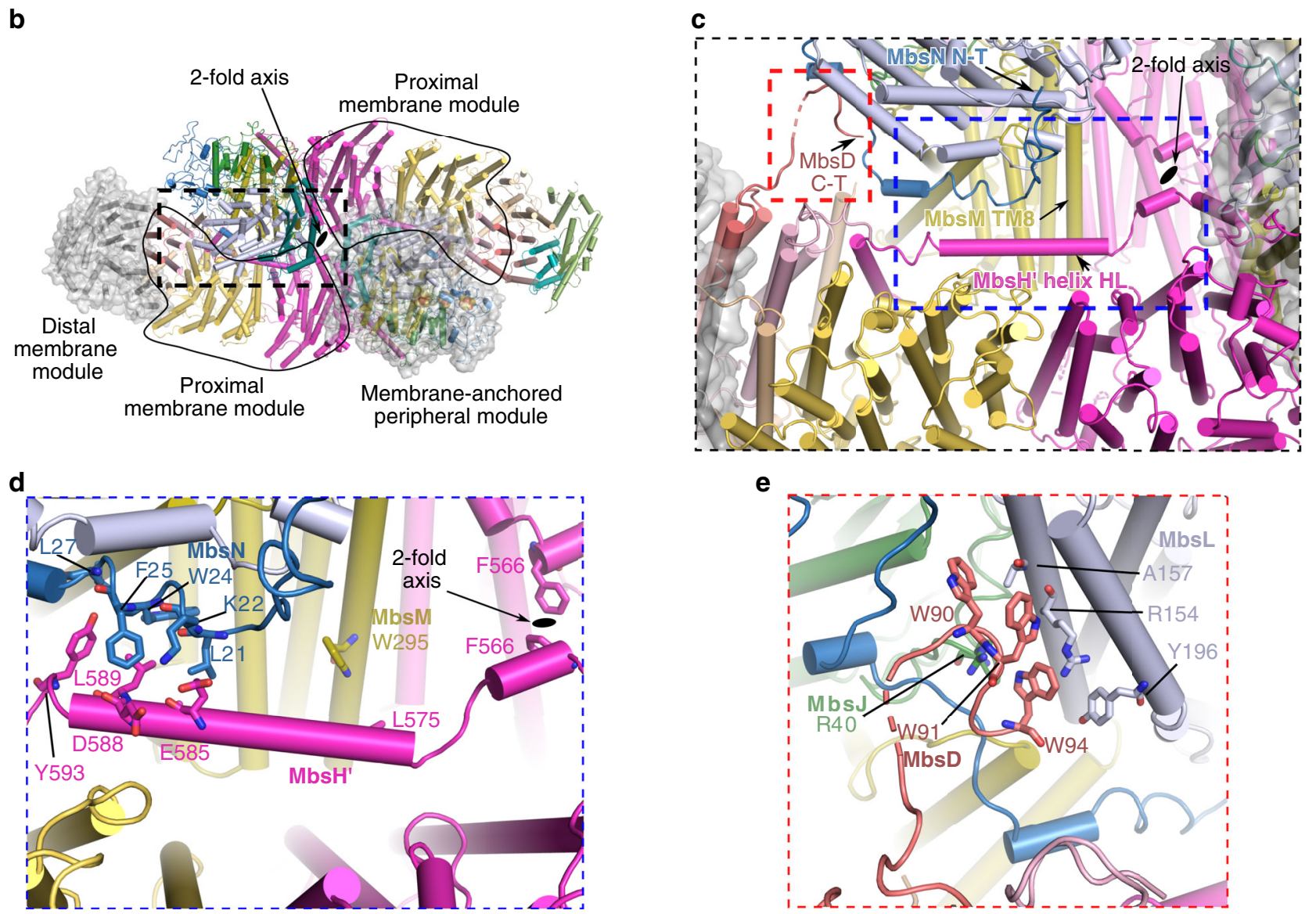

Fig. 2 The architecture of the dimeric MBS complex. a Side (left) and top cytoplasmic views (right) of an MBS monomer. The central proximal membrane module is flanked by the distal membrane module and the membrane-anchored peripheral module, which are further highlighted by transparent surface views. $\mathbf{b}$ A top view showing the assembly of the dimeric MBS complex with the three different modules indicated as in (a). c A zoomed view of the dashed rectangle in (b). The dimer interface is composed of two regions, one involving the Helix $\mathrm{HL}$ of $\mathrm{MbsH}^{\prime}$ (dashed blue box) and the other involving the elongated C-terminal tail of MbsD (dashed red box). d A close-up view of the dashed blue box in (c). e A close-up view of the dashed red box in (c). Residues contributing to the dimer interface are shown as sticks in (d) and (e).

In the distal membrane module of MBS, MbsA at the far end contains two TMHs and a following ferredoxin-like domain and binds to the three 3-TMH subunits MbsB, C, and TMH3-6 of MbsE, which in turn are attached to MbsD of the proximal membrane module (Supplementary Fig. 3a). Notably, MbsB, C, and $\mathrm{E}$ contain three conserved regions that are rich in charged/ polar residues and run across the interior of the membrane arm (Supplementary Fig. 4e). In addition, the conserved MbsC P83 introduces a break in the middle of the membrane of $\mathrm{MbsC}$ TMH3. These features are conserved in the $\mathrm{Na}^{+}$-translocation path in $\mathrm{MBH}$ and in Mrp, but not in complex I as complex I does not translocate $\mathrm{Na}^{+}$ions (Fig. 3a, Supplementary Fig. 4d) ${ }^{6,34}$. This suggests a $\mathrm{Na}^{+}$-translocation path within this sub-region. This together with the adjacent $\mathrm{H}^{+}$path $(\mathrm{Pc})$ likely forms the $\mathrm{Na}^{+} / \mathrm{H}^{+}$antiporter module in the distal end of the membrane arm (Fig. 3c).

During the review of this manuscript, the structure of Mrp antiporter was published ${ }^{35}$. The structure enables a comparison between MBS and Mrp to obtain direct evolutionary insights between them (Fig. 4a). All subunits of the $\mathrm{Mrp} \mathrm{Na}+/ \mathrm{H}^{+}$ 
a

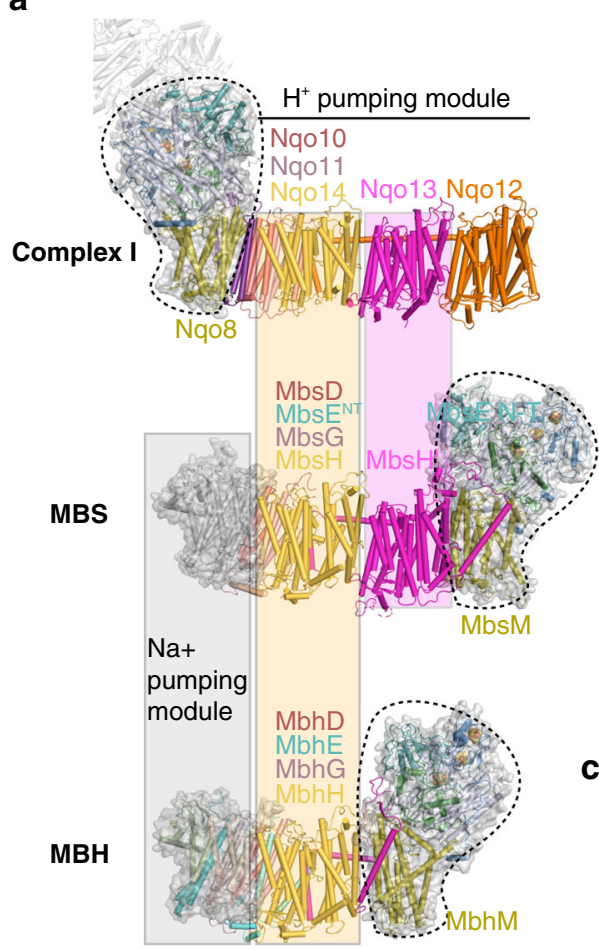

b

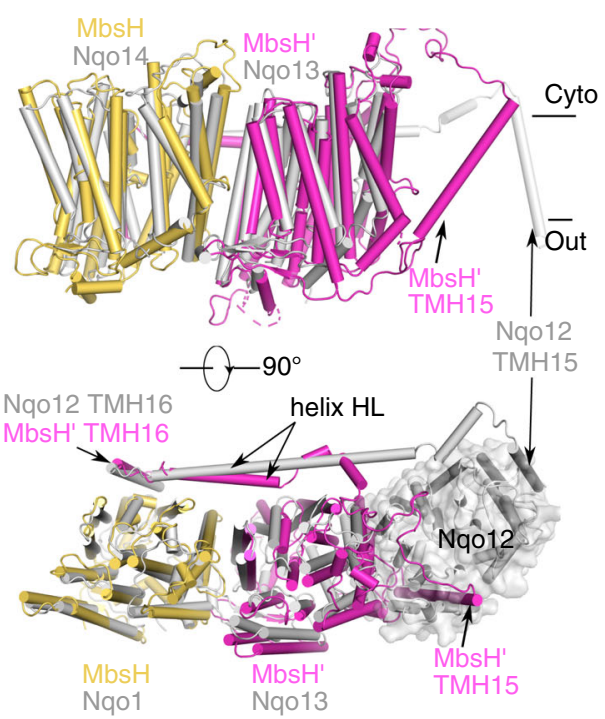

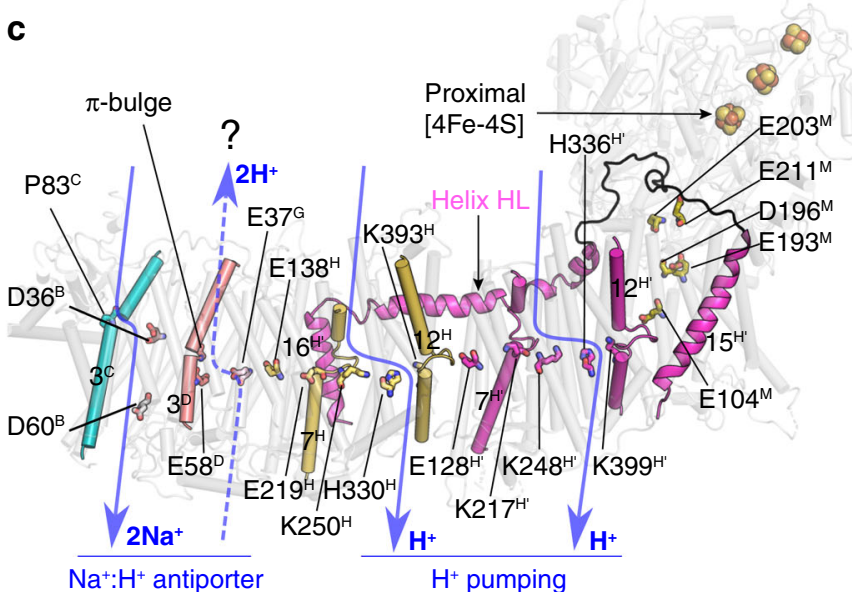

Fig. 3 Assembly of the modular architecture of MBS and comparison with complex I and MBH. a Complex I (T. thermophilus; PDB ID 4HEA) is lined up with MBS and MBH (P. furiosus; PDB ID 6CFW) via their respective proton-pumping subunit Nqo14, MbsH, and $\mathrm{MbhH}$. The alignment detail is shown in Fig. S4. The complexes are shown separately for clarity. All complexes have a similar peripheral arm (dashed outline) anchored either to the left end

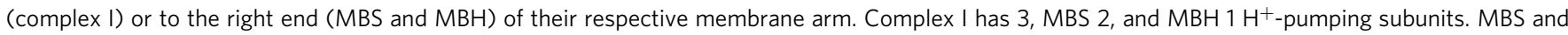
$\mathrm{MBH}$, but not complex I, have a Na+ translocation module. b Superimposition of the two antiporter-like subunits of $\mathrm{MBS}\left(\mathrm{MbsH}, \mathrm{MbsH}^{\prime}\right)$ and complex I (Nqo14, Nqo13), by aligning MbsH with Nqo14 as in (a). Top, side view with complex I Nqo12 TMH1-14 hidden; bottom, top view with complex I Nqo12 TMH1-14 shown as cartoon and marked by the transparent surface. Note that the length of helix HL is evolved to match the number of antiporter-like subunits present in MBS and complex I. c The potential $\mathrm{Na}^{+}$and $\mathrm{H}^{+}$translocation paths in MBS. The $\mathrm{Na}^{+}$path and the adjacent $\mathrm{H}^{+}$path together form the potential $\mathrm{Na}^{+} / \mathrm{H}^{+}$antiporter module in the distal end of the membrane arm. The question mark above the proton path in the antiporter module indicates the tentative nature of the assignment. In the three $\mathrm{H}^{+}$paths, key residues conserved with complex I are shown as sticks. In the $\mathrm{Na}^{+}$path, key residues conserved with $\mathrm{MBH}$ and $\mathrm{Mrp}$ are shown as sticks. A series of charged and protonatable residues extend from $\mathrm{MbsM}$ to $\mathrm{MbsH}$ and $\mathrm{MbsH}$ to form the charged axis across the membrane interior.

antiporter have homologous counterparts in MBS (Supplementary Table 2). Consequently, the MBS membrane arm (excluding MbsM, which anchors the peripheral arm) shares a conserved architecture with Mrp (Fig. 4a). It is clear that the Mrp structure lacks the membrane-anchored peripheral arm and supports the modular evolution of respiratory complexes ${ }^{31}$. The emergence of MBS and MBH was likely a result of the combination of the Mrp antiporter and a peripheral module related to a cytoplasmic [NiFe] hydrogenase. MBH exhibits loss of the MrpA homolog resulting in fewer proton pumps while its peripheral arm retains the proton reduction activity. In contrast, MBS keeps all the Mrp homologs while its peripheral arm shows concomitant changes in the peripheral module to enable catalysis of the reduction of polysulfide rather than protons (Fig. 4b). Conceivably, both MBS and $\mathrm{MBH}$ are much more efficient in energy conservation as the redox reaction is now physically coupled with the ion translocation across the membrane.

A three-loop system bridges the electron transfer site and the ion-pumping membrane arm. The architecture of the MBS cytoplasmic module closely resembles those of $\mathrm{MBH}$ and complex I (Supplementary Fig. 5). In MBS, a large hydrophobic chamber, similar in size to those in $\mathrm{MBH}$ and complex $\mathrm{I}$, is formed by cytoplasmic MbsL, MbsJ, and their membrane anchor MbsM (Figs. 5a-c, 6a). The openings of the MBS and complex I hydrophobic chamber are wider than that of $\mathrm{MBH}$ allowing entry of hydrophobic substrates (polysulfide and quinone; Fig. 5d). The end of this chamber in MBS coincides with the binding site of the quinone headgroup defined by Nqo4 H38 and Y87 in complex I 


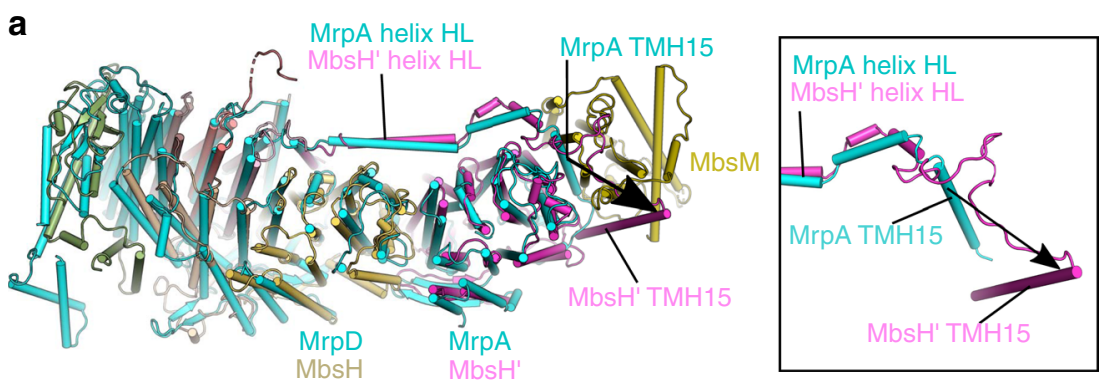

b

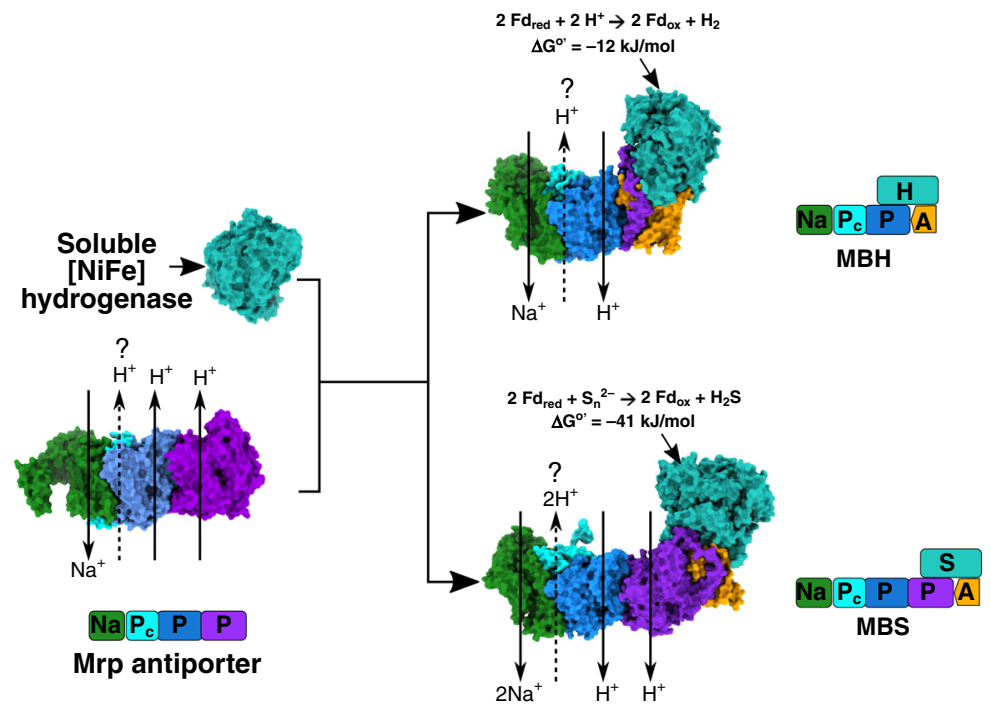

Fig. 4 Evolutionary relationship of Mrp, MBH, and MBS. a Comparison of the MBS membrane arm with the Mrp structure (PDB ID: 6Z16). The structures are aligned by superimposing MbsH and MrpD. The MBS subunits are colored as Fig. 1c and the Mrp is in cyan. Mrp structure is similar to MBS, with the conserved helix $\mathrm{HL}$ holding the two antiporter-like subunits in both complexes (MrpA and MrpD; MbsH and MbsH'). Notably, the MbsH' $\mathrm{TMH}^{\prime} 5$ immediately preceding helix $\mathrm{HL}$ moves a large distance, as compared to MrpA TMH15, to pack tightly against MbsM in order to accommodate MbsM in the MBS structure (see inset panel to the right). b A possible evolutionary pathway from Mrp to MBH and to MBS. Mrp antiporter: PDB ID 6Z16; cytoplasmic [NiFe] hydrogenase: PDB ID 2FRV; MBH: PDB ID 6CFW. The question mark above the proton path in each antiporter module indicates the tentative nature of the assignment. Shown above the MBH and MBS structures are the reductive reactions that they catalyze and the associated free energy changes. Located around each complex is the respective modular architectures of the Mrp-derived respiratory complexes. These modules are: Na (green), sodium ion path; $\mathrm{P}$ (blue and purple), $\mathrm{H}^{+}$path(s) formed within a single subunit; $\mathrm{Pc}$ (cyan), composite $\mathrm{H}^{+}$path formed by multiple small subunits; A (orange), membrane anchor for the peripheral arm; $\mathrm{H}$ (turquoise), peripheral arm for proton reduction; $\mathrm{S}$ (turquoise), peripheral arm for polysulfide reduction.

(Fig. 5e $)^{1}$. However, these two residues for quinone headgroup binding and also for protonation in complex I are absent in MBS (Fig. 5f). Moreover, MbhL E21, the key residue for proton reduction in the $\mathrm{MBH}$ complex, is also absent in $\mathrm{MBS}^{6}$. These suggest a unique catalytic mechanism for MBS despite similar modular overall architecture, as we will cover in the next section.

This hydrophobic chamber containing the catalytic site of MBS in the peripheral arm is defined by three loops originating from

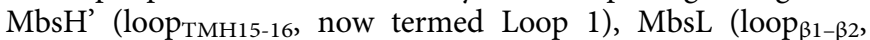

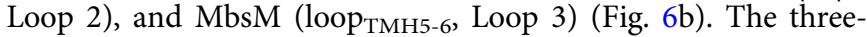
loop cluster connects the electron-transferring peripheral arm to the ion-pumping membrane arm and is strikingly similar in the three complexes (Fig. 6b) ${ }^{1-3,6}$. In MBS, Loop 1 via TM15 and TM16 of MbsH' connects to its horizontal helix HL that spans two proton pumps (MbsH and MbsH') (Supplementary Fig. 4a). Similarly in MBH, Loop 1 connects TM1 and TM2 of MbhI to its shorter helix HL that spans a single proton pump (MbhH). In complex I, Loop 1 and its preceding TM1 of Nqo7 locate the conserved membrane-anchored peripheral module to a different site of the membrane arm via TM2-3 of Nqo7 while the much longer helix HL of Nqo15 that spans three proton pumps (Nqo12, 13 , and 14) is similar to MBS and MBH (Supplementary Fig. 4a).
A central charged axis in MBS extending from the three loops across the membrane arm in parallel to helix HL is also found in both complex I and $\mathrm{MBH}$ (Figs. 3c, 6c) ${ }^{1-3,6,32}$. Clearly, the structural elements in $\mathrm{MBH}$ and complex I necessary for energy conservation are maintained in MBS. Therefore, we suggest that Loop1 and its preceding TM are a key structural feature that has been conserved across evolution in order to sustain the fundamental function of coupling the redox reaction in the peripheral arm with ion translocation in the membrane arm.

The peripheral arm of MBS contains a uniquely coordinated [4Fe-4S] cluster. In MBS, the three [4Fe-4S] clusters form an efficient electron transfer pathway to the chamber from $\mathrm{Fd}$ (Fig. 1c inset) ${ }^{36}$. MbsN coordinates the distal and the medial [4Fe-4S] clusters (relative to the catalytic chamber; Supplementary Fig. 6a). The proximal (p-) $[4 \mathrm{Fe}-4 \mathrm{~S}]$ cluster in MBS and complex I is at the top of the hydrophobic chamber (Supplementary Fig. 6b) but, in stark contrast to that in complex I (and $\mathrm{MBH})$, the MBS $\mathrm{p}-[4 \mathrm{Fe}-4 \mathrm{~S}]$ is coordinated by only three rather than the expected four cysteines (C28, C93 and C123 of MbsJ; Fig. 6d). 


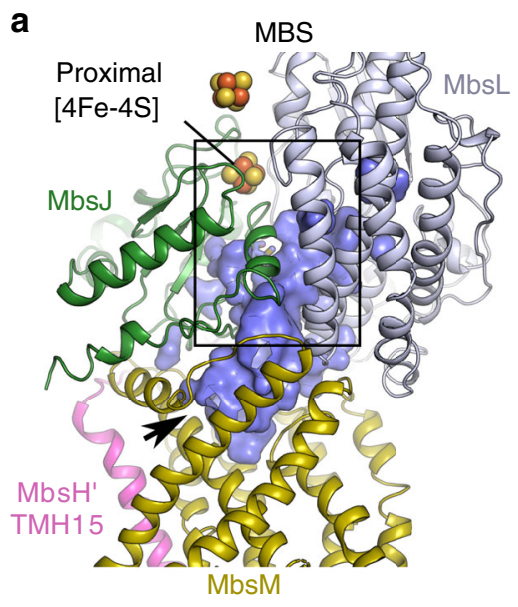

d

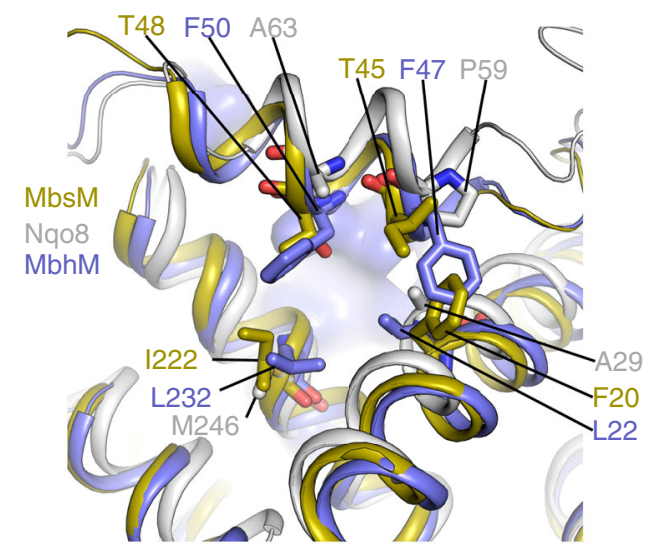

b

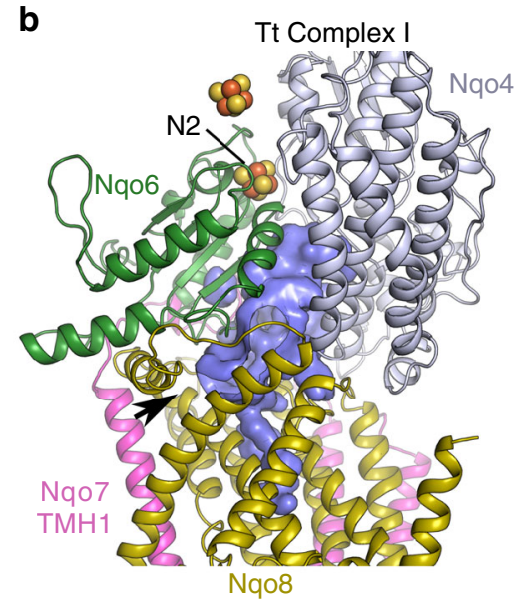

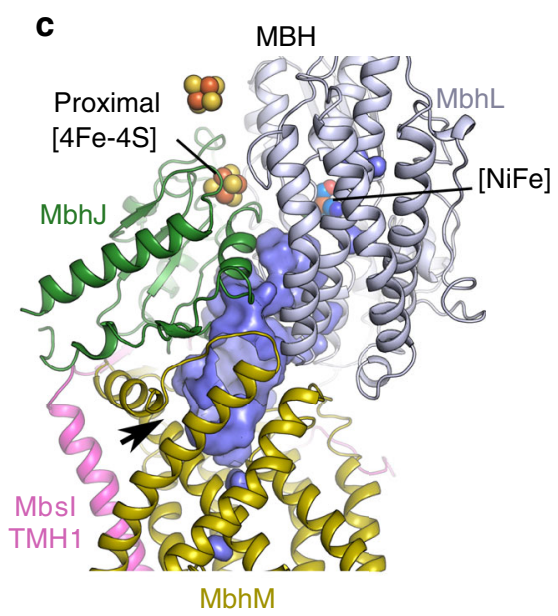

e Proximal $4 \mathrm{Fe}-4 \mathrm{~S}]$

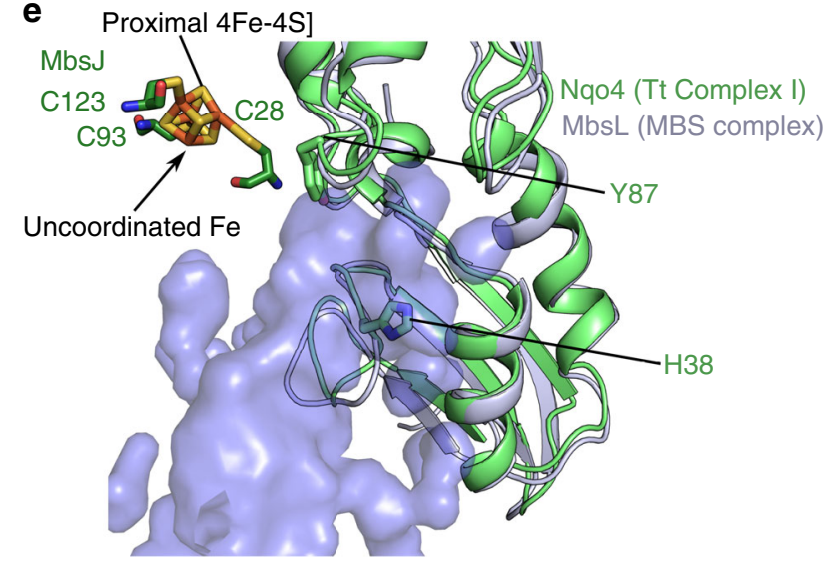

f



Fig. 5 A comparison of the chamber at the active sites of MBS, MBH and complex I. The structures were overlaid based on MbsM, Nqo8, and MbhM and are shown individually. The chamber is similarly formed at the interface between the peripheral arm and its membrane anchor. The black arrow indicates the opening of the chamber proposed as the entrance of quinone in complex I studies. a MBS. b Complex I (PDB ID 4HEA). c MBH (PDB ID 6CFW). d A comparison of the chamber entry between $\mathrm{MBS}, \mathrm{MBH}$, and complex I indicate a wider opening in MBS and complex I than that in MBH. $\mathrm{e}$ Zoomed view of the end of the active site chamber, outlined as the boxed region in (a). The aligned Nqo4 of Tt complex I is shown as a green cartoon where two residues ( $\mathrm{H} 38$ and $\mathrm{Y} 87$ ) proposed for the binding of the quinone headgroup are shown as sticks. $\mathbf{f}$ Sequence alignment of MbsL with its counterparts in different respiratory complexes (Nqo4 of Tt complex I, NdhH of NDH complex, MbhL of MBH complex I). Residues equivalent to Nqo4 H38 and Y87, proposed for quinone headgroup binding and protonation in complex I, and MbhL E21, the key residue for proton reduction in MBH complex, are all absent in MBS.

The different functions of the $\mathrm{p}$-[4Fe-4S] clusters in these enzymes (Fig. 5b) are determined by a conserved structural motif containing a short $\mathrm{N}$-terminal $\alpha$-helix, a $\beta$-strand, a variable loop with two cysteines and another short $\alpha$-helix (in MbsJ for MBS complex) (Supplementary Fig. 6b, c). In MBH and cytosolic $[\mathrm{NiFe}]$ hydrogenases ${ }^{6,37}$, the two cysteines are two residues apart and ideally positioned to both coordinate the proximal cluster, which donates electrons to the $\mathrm{NiFe}$-catalytic site. In complex I, the two cysteines (C45 and $\mathrm{C} 46$ in Nqo6) are adjacent to each other enabling sequential dissociation upon proximal cluster reduction, which has been proposed to facilitate electron and proton transfer to the quinone although this is under debate ${ }^{16,38,39}$. In MBS, this structural motif is three residues shorter than it is in MBH, leaving one of the cysteines (MbsJ C25) no longer able to participate in cluster coordination (Fig. 6d, Supplementary Fig. 6c). Hence, one iron atom of the p-4Fe-4S] cluster in MBS has non-cysteinyl coordination (Supplementary Fig. 2j) and therefore catalytic potential. Besides the three [4Fe$4 \mathrm{~S}]$ clusters, $\mathrm{MBH}$ has an additional proton-reducing [NiFe]-site, which is coordinated by four cysteines of MbhL (Supplementary Fig. 6d) ${ }^{6,40}$. Of these four cysteines, two are not present in MBS, leaving the two conserved residues (C85 and C385 in MbsL) adjacent to the proximal cluster (Fig. 7a). Complex I has lost all four, supporting the stepwise evolution of MBS and complex I by 
a

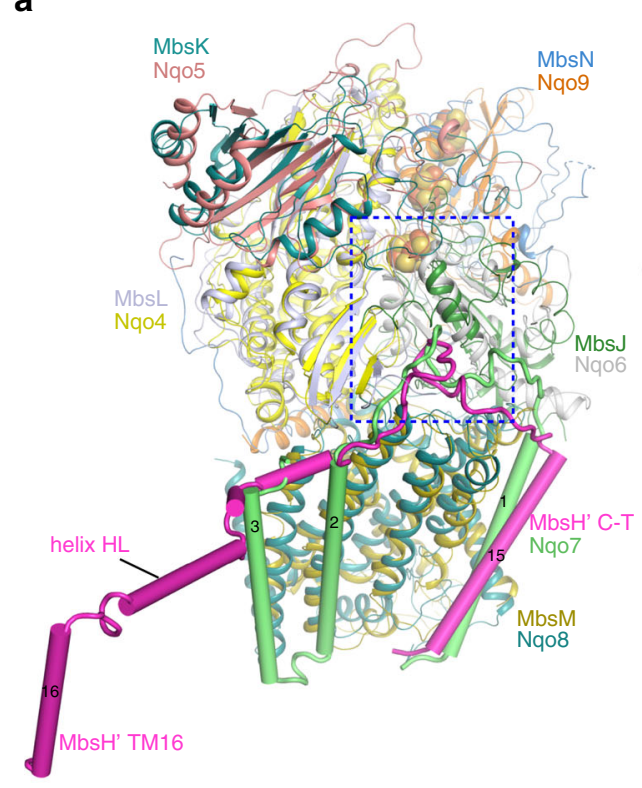

C

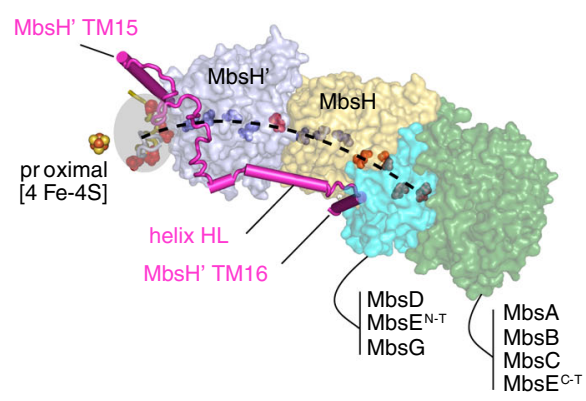

b

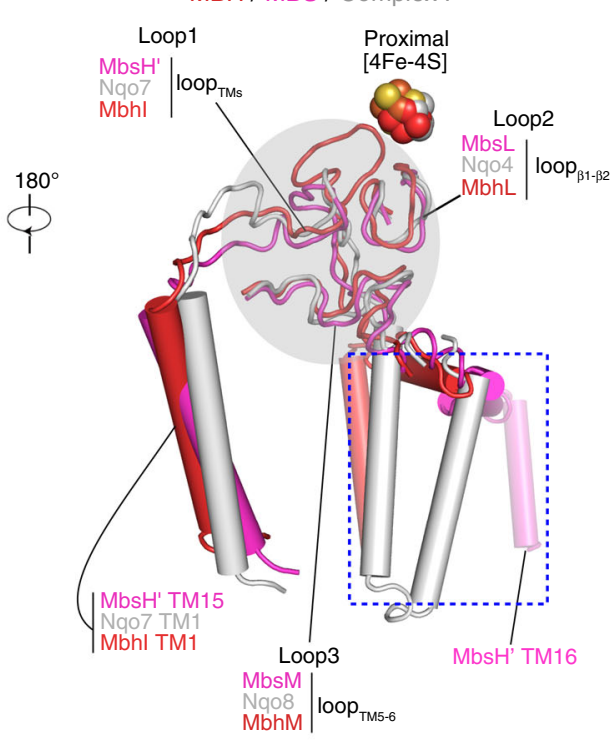

d

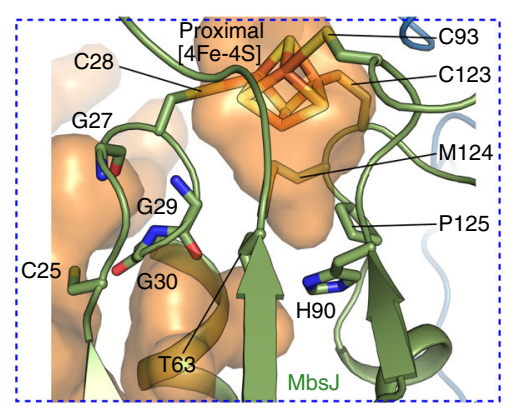

Fig. 6 Membrane-anchored peripheral module of MBS. a Superimposition of the membrane-anchored peripheral modules of MBS and complex I by aligning MbsL with Nqo4. The aligned regions are shown in cartoons and colored as the text labels. Structural elements that bridge the peripheral module to the $\mathrm{H}^{+}$-pumping module (complex I Nqo7 and MBS MbsH' TMH15-16, more details in Supplementary Fig. 4a) are shown as cylinders. b Between the peripheral arm and membrane arm are three interfacial loops, highlighted in gray shade, that are remarkably similar in MBH (red), MBS (magenta) and

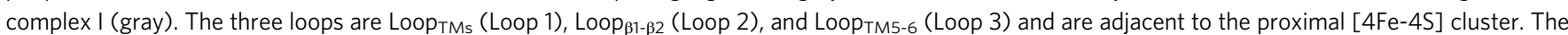
C-terminal TMs following Loop 1 (boxed in the dashed blue square) are least similar among the three-loop systems of MBH, MBS, and complex I. c The three-loop system (gray shade) is linked to the membrane ion translocation module in the semi-transparent surface through the C-terminal TMs following Loop 1. The dashed curve marks the central charge axis, a chain of red (negatively charged residues) and blue spheres (positively charged residues) spanning across the membrane arms. $\mathbf{d}$ Zoomed view of the area boxed with dashed blue square in (a) showing the coordination of the proximal [4Fe-4S]. MbsJ residues C28, C93 and C123 coordinate three irons of proximal [4Fe-4S] cluster, but MbsJ C25 is too far to coordinate the remaining iron. The semitransparent orange surfaces show potential solvent-accessible chambers around the proximal cluster.

sequential loss of the four cysteines coordinating the [NiFe] center in $\mathrm{MBH}^{31,41}$.

In MBS, two potential polysulfide-reducing sites are close $(\sim 12 \AA)$ to the p-[4Fe-4S] cluster: MbsJ C25 and MbsL C85/ C385 (Fig. 7a). However, they are not involved in catalysis as the C25A MbsJ and the C85A/C385A MbsL mutant enzymes had comparable catalytic activities using the model substrate dimethyltrisulfide (DMTS) to that of the wild-type (WT) enzyme (1.8 \pm 0.4 units/mg) (Fig. $7 \mathrm{~b}$ ). Cluster interconversion was used to provide evidence that the $\mathrm{p}-[4 \mathrm{Fe}-4 \mathrm{~S}]$ cluster of MBS is itself reducing polysulfide, and doing so with a specific role for its unique non-cysteinyl coordinated Fe atom. The WTenzyme was treated with potassium ferricyanide, a wellestablished procedure to remove non-cysteinyl coordinated $\mathrm{Fe}$ atom from an $[4 \mathrm{Fe}-4 \mathrm{~S}]$ cluster to generate a [3Fe-4S] cluster ${ }^{21}$. This caused an $\sim 98 \%$ loss of activity (Fig. $7 \mathrm{~b}$ ). Subsequently incubating the enzyme under reducing conditions with ferrous iron, conditions that are known to restore the [4Fe-4S] cluster from a [3Fe-4S] cluster, the MBS activity was restored to its original value (Fig. $7 \mathrm{~b}$ ).

Further evidence for the proposed catalytic p-[4Fe-4S] cluster came from EPR spectroscopy. MBS from $S^{0}$-grown cells gave rise to an intense free-radical EPR signal $(g=2.00)$ unaffected by chemical reduction (Supplementary Fig. 7a) but this was a purification artifact because the enzyme from non- $S^{0}$ grown cells (in which transcription of the genes encoding MBS was controlled by a non-regulated promoter) did not exhibit this EPR signal (Supplementary Fig. 7b). Chemical reduction of MBS did lead to the appearance of a broad EPR signal $(g \sim 1.94)$, consistent with three interacting and reduced [4Fe-4S] clusters (Supplementary Fig. 7b). After treatment with excess ferricyanide, the inactive enzyme (Fig. 7b) exhibited a complex EPR signal indicative of an oxidized $S=1 / 2[3 \mathrm{Fe}-4 \mathrm{~S}]^{+}$cluster $(g=2.09$, Supplementary Fig. $7 c)^{21}$, supporting the conclusion that the $\mathrm{p}$-[4Fe-4S] cluster is catalytic and that activity is reversibly lost upon removal of its unique iron. Importantly, chemically reduced P. furiosus $\mathrm{MBH}$ 
a

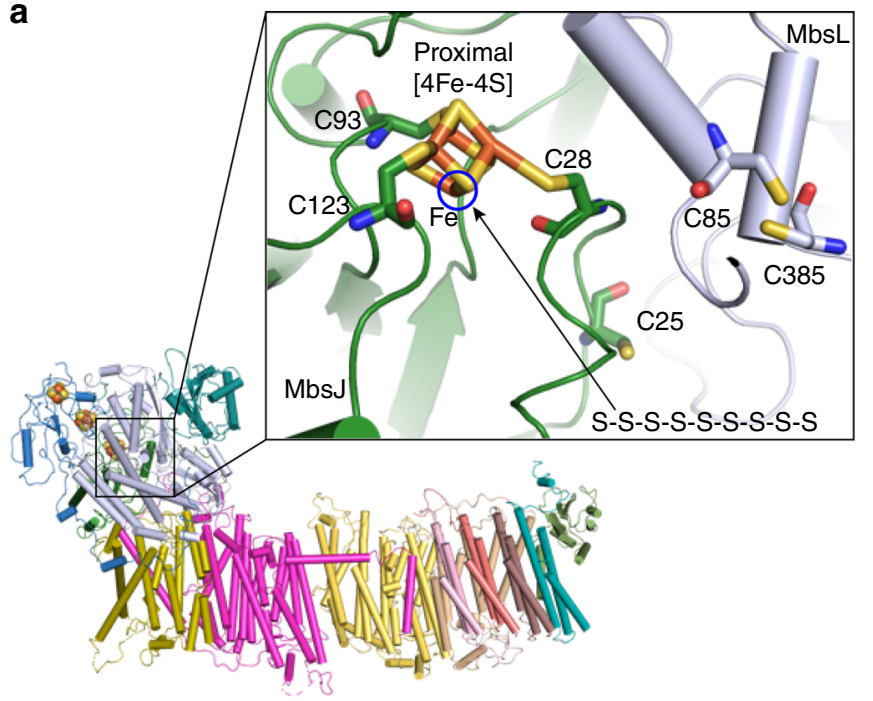

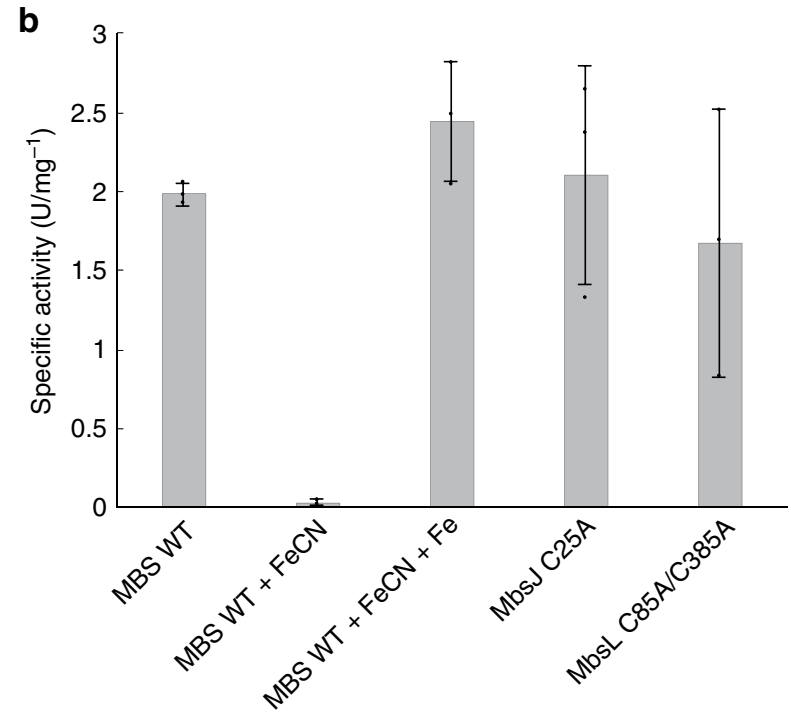

C


d

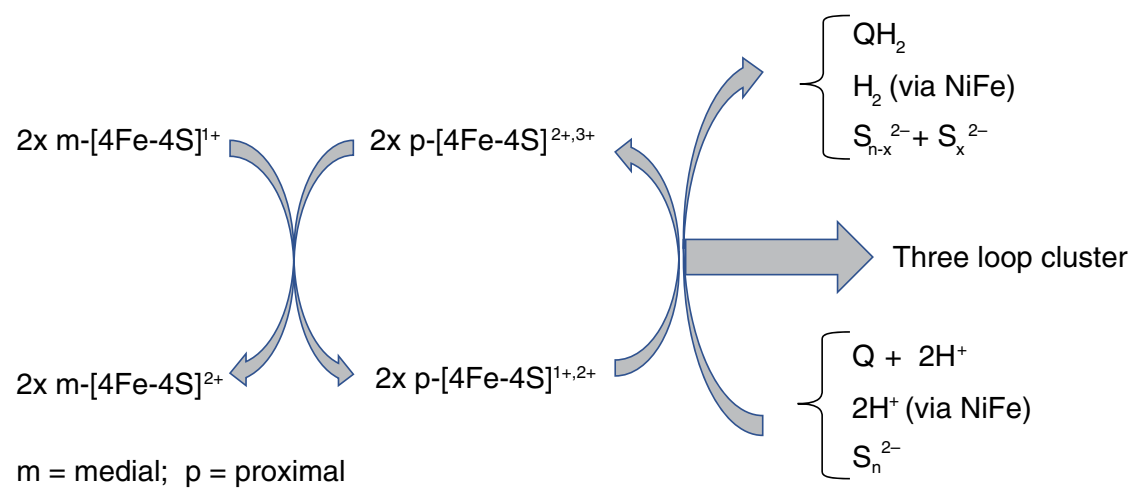


Fig. 7 MBS catalyzed polysulfide reduction mechanism. a Cysteine residues within electron tunneling distance of the proximal [4Fe-4S] cluster. b Polysulfide reduction activity assay of purified WT and mutant C-MBS subcomplexes, demonstrating that MbsJ C25 and MbsL C85 and C385 are not involved in catalysis. Error bars are centered from the average value and represent standard deviation obtained using technical triplicate samples. c Proposed catalytic mechanism of polysulfide reduction for the MBS complex. d A sketch summarizing energy transduction in complex I, MBH, and MBS.

gave rise to an EPR signal (Supplementary Fig. 7d) similar to that of reduced MBS (Supplementary Fig. 7b), but when treated with ferricyanide under the same conditions used for MBS, no activity was lost and the MBH sample was EPR silent, consistent with the presence of only oxidized [4Fe-4S] clusters (Supplementary Fig. 7e). Unlike MBS, the p-[4Fe-4S] cluster of $\mathrm{MBH}$ is only involved in electron transfer and has full cysteinyl coordination, hence it is not susceptible to inactivation due to iron loss and oxidative cluster conversion.

The proximal [4Fe-4S] cluster uses a novel, ancestral mechanism for sulfur reduction. The reduction of polysulfide by the p-[4Fe-4S] cluster in the hydrophobic pocket of MBS (Fig. 7a) is the first example in a biological system of reduction of an inorganic disulfide bond without the release of $\mathrm{H}_{2} \mathrm{~S}$ (Eqs. 2 and 3, c.f. Eq. 5). Three types of enzymes use iron-sulfur clusters to indirectly facilitate disulfide bond cleavage; heterodisulfide reductase ${ }^{42}$, hydrogenase processing protein $\mathrm{HydD}^{43}$, and ferredoxin thioredoxin reductase (FTR) ${ }^{44}$. Based in part on FTR, we propose a new mechanism for the reduction of an internal disulfide (which could be either inorganic or organic) by MBS (Fig. 7c). The unique $\mathrm{Fe}$ atom of its $\mathrm{p}-[4 \mathrm{Fe}-4 \mathrm{~S}]^{2+}$ cluster binds polysulfide $\left(\mathrm{S}_{\mathrm{n}}{ }^{2-}\right)$, accepts an electron from the medial (m-) $[4 \mathrm{Fe}-4 \mathrm{~S}]^{1+}$ cluster (Fig. 1c) and catalyzes the two-electron reduction of an internal disulfide, generating two smaller polysulfides $\left(\mathrm{S}_{\mathrm{n}-\mathrm{x}}{ }^{2-}\right.$ and $\left.\mathrm{S}_{\mathrm{x}}{ }^{2-}\right)$ and the superoxidized form of $\mathrm{p}-[4 \mathrm{Fe}-4 \mathrm{~S}]^{3+}$. As in FTR, the reduction of the $\mathrm{p}-[4 \mathrm{Fe}-4 \mathrm{~S}]^{2+}$ cluster actually leads to its superoxidation because two electrons are used to reduce the disulfide. Moreover, the cluster must be in the $3+$ (rather than $2+$ ) state to bind the two product polysulfides at the unique Fe site (Fig. 7c). The catalytic cycle is completed by reduction of $\mathrm{p}-[4 \mathrm{Fe}-4 \mathrm{~S}]^{3+}$ by a second electron from $\mathrm{m}-[4 \mathrm{Fe}-4 \mathrm{~S}]^{1+}$ (after its reduction by the distal $[4 \mathrm{Fe}-$ $4 \mathrm{~S}]^{1+}$ cluster) and release of the two smaller polysulfides. This is the simplest mechanism yet proposed for the reduction of an S-S bond in either an inorganic or organic sulfide and is the first not requiring either protons or amino acid residues ${ }^{42-44}$. This reaction is clearly of fundamental significance in iron and sulfur-rich hydrothermal vent environments of the early earth and possibly in the origin of early life ${ }^{21}$, as well as in the subsequent evolution and expansion of iron-sulfur cluster functionality in the diverse array of modern-day iron-sulfur proteins ${ }^{22,45}$.

That catalysis by MBS does not require water or protons (Fig. 7c) is consistent with a highly hydrophobic pocket and the absence in MbsL of the well-defined proton transfer pathway to the $\mathrm{NiFe}$ site found in $\mathrm{MbhL}$ and other hydrogenases ${ }^{6}$. The requirement for binding two polysulfides (Fig. $7 \mathrm{~b}$ ) is superoxidation of the $\mathrm{p}$-[4Fe-4S] cluster $(3+)$, which is facilitated by the hydrophobic site, analogous to superoxidation of hydrophobic [4Fe-4S] clusters in high potential iron-sulfur proteins (HiPIPs) ${ }^{46}$. The spontaneous breakdown of tri- and disulfides to release $\mathrm{H}_{2} \mathrm{~S}$ does require protons (Eq. 4) and must occur in the cytoplasm rather than the hydrophobic catalytic site (Fig. 7c). The di- and trisulfides produced by MBS do not generate $\mathrm{H}_{2} \mathrm{~S}$ (or $\mathrm{HS}^{-}$), are stable in the hydrophobic pocket (where there are no protons or water), and $\mathrm{H}_{2} \mathrm{~S}$ is only generated when they are released from the enzyme (Fig. $7 \mathrm{c}$ ).

\section{Discussion}

The standard state $\Delta \mathrm{G}(\mathrm{pH} 7.0)$ of the electron transfer reactions for MBS $\left(41 \mathrm{~kJ} / \mathrm{mol} / 2 \mathrm{e}^{-}\right)$is more than twice that available to
$\mathrm{MBH}\left(12 \mathrm{~kJ} / \mathrm{mol} / 2 \mathrm{e}^{-}\right)$, and half of that available to complex I $\left(81 \mathrm{~kJ} / \mathrm{mol} / 2 \mathrm{e}^{-}\right)^{9}$, consistent with the pumping of two, one and four protons, respectively $1,2,7,8,47$. The MBS structure confirms that the transduction of redox energy into the spatially separated translocation of ions involves the three-loop cluster ${ }^{1-3,31,48}$ and a central charged axis ${ }^{1-3,7,32}$ (Fig. 6b, c), all highly conserved across evolution. The proposed MBS mechanism also shows that there are key fundamental differences in spite of a common overall means of energy transduction. In MBS, oxidation of the reduced $\mathrm{m}-[4 \mathrm{Fe}-4 \mathrm{~S}]^{1+}$ cluster leads to superoxidation of the oxidized $\mathrm{p}$ $[4 \mathrm{Fe}-4 \mathrm{~S}]^{2+}$ cluster $\left(2^{+} / 3^{+}\right.$redox couple) but in $\mathrm{MBH}$ and complex I to reduction of the oxidized $\mathrm{p}$-[4Fe-4S $]^{1+}$ cluster $\left(2^{+} / 1^{+}\right.$ redox couple; Fig. 7c). Hence the loss of the $\mathrm{NiFe}$ site of an ancestral MBH through evolution generated a highly hydrophobic cavity enabling both MBS and complex I to use the p-[4Fe-4S] cluster to directly interact with and reduce hydrophobic substrates, albeit using different charge states of the cluster (Fig. 7d).

It is not clear how a change in the redox status of the $m-[4 \mathrm{Fe}-$ $4 \mathrm{~S}$ ] cluster is ultimately coupled to a change in the dynamics of the three-loop cluster, nor how the different amounts of free energy released $\left(12-81 \mathrm{~kJ} / \mathrm{mol} / 2 \mathrm{e}^{-}\right)$lead to corresponding differences in the proton motive force that is generated (1-4 protons). It is interesting to speculate that MBS might conserve more (or less energy) if one proton pump ( $\mathrm{MbsH}^{\prime}$ or equivalent, see Fig. 3c) were added (or inactivated, by mutagenesis) and that the cell yield of $P$. furiosus growing on $S^{0}$ would be correspondingly higher (or lower). Such experiments are in progress. On a more fundamental level, the question yet to be answered is, as illustrated in Fig. 7 d, how does $\mathrm{m}-[4 \mathrm{Fe}-4 \mathrm{~S}]^{1+}$ oxidation coupled to a change in the conformation of the three-loop cluster lead to the conservation of different quantities of energy in these three types of the respiratory complex? In any event, the simple aprotic and abiotic catalytic mechanism of S-S bond reduction by MBS might be a modern remnant of an iron-sulfur world ${ }^{21}$ as well as the precursor to more complex mechanisms involving iron-sulfur clusters $^{42-44}$ and those now found in the aerobic world, such as the reduction of quinones by complex I.

\section{Methods}

Purification of MBS. The MBS holoenzyme (S-MBS) was solubilized and purified anaerobically from Pyrococcus furiosus strain MW0491, in which a His -tag had been engineered at the $\mathrm{N}$ terminus of the MbhJ subunit ${ }^{19}$. Frozen cells were lysed in $25 \mathrm{mM}$ sodium phosphate, $\mathrm{pH} 7.5$, containing $1 \mathrm{mM}$ DTT and $50 \mu \mathrm{g} / \mathrm{ml} \mathrm{DNase} \mathrm{I}$ ( $5 \mathrm{~mL}$ per gram of frozen cells). After stirring for one hour, the cell-free extract was centrifuged at $100,000 \times \mathrm{g}$ for one hour. The supernatant was removed and the membranes were washed twice using $50 \mathrm{mM}$ EPPS buffer, $\mathrm{pH}$ 8.0, containing $5 \mathrm{mM} \mathrm{MgCl}_{2}, 50 \mathrm{mM} \mathrm{NaCl}, 10 \%$ (v/v) glycerol, $1 \mathrm{mM} \mathrm{DTT}$, and $0.1 \mathrm{mM} \mathrm{PMSF}$. The membrane pellet was collected by ultracentrifugation at $100,000 \times \mathrm{g}$ for one hour after each wash step. The washed membranes were resuspended in $50 \mathrm{mM}$ Tris- $\mathrm{HCl}, \mathrm{pH}$ 8.0, containing $5 \mathrm{mM} \mathrm{MgCl}_{2}, 50 \mathrm{mM} \mathrm{NaCl}, 5 \%$ (v/v) glycerol, $1 \mathrm{mM}$ DTT, and $0.1 \mathrm{mM}$ PMSF. MBS was solubilized by adding $\mathrm{n}$-dodecyl- $\beta$-D-maltoside (DDM, Inalco) to $3 \%(\mathrm{w} / \mathrm{v})$ followed by incubation at $4{ }^{\circ} \mathrm{C}$ for 16 hours. The solubilized membranes were centrifuged at $100,000 \times g$ for $1 \mathrm{~h}$. The supernatant was applied to a $5 \mathrm{~mL}$ His-Trap crude FF Ni-NTA column (GE Healthcare) while diluting it 10 -fold with buffer A ( $25 \mathrm{mM}$ sodium phosphate, $300 \mathrm{mM} \mathrm{NaCl}, \mathrm{pH} 7.5$, containing $1 \mathrm{mM}$ DTT and $0.03 \%$ DDM). The column was washed with 10 column volumes of buffer A and the bound protein was eluted with a 20 -column volume gradient from 0 to $100 \%$ buffer B (buffer A containing $500 \mathrm{mM}$ imidazole). The eluted protein was further purified by applying it to a 1-mL His-Trap HP Ni-NTA column (GE Healthcare) while diluting it 5-fold with buffer A. A 30-column volume gradient from 0 to $100 \%$ buffer B was used to elute the bound protein. The MBS sample was concentrated and further purified using a Superose 6 10/300 GL column (GE Healthcare) equilibrated with $50 \mathrm{mM}$ Tris- $\mathrm{HCl}, \mathrm{pH} 8.2$, containing
} $300 \mathrm{mM} \mathrm{NaCl}, 2 \mathrm{mM}$ sodium dithionite, and $0.03 \% \mathrm{DDM}$. 
P. furiosus strain construction. The strains used in this study are summarized in Supplementary Table 3 . The genetically tractable $P$. furiosus strain COM1 was used for the genetic manipulation of $\mathrm{MBS}^{49}$. An insertion cassette was amplified using overlapping $\mathrm{PCR}^{50}$. The upstream flanking region (UFR), which also contained the selection marker $\left(\mathrm{P}_{\mathrm{gdh}}-\mathrm{pyrF}\right)$ and the promoter of the gene encoding the S-layer protein $\left(\mathrm{P}_{\text {slp }}\right)$, and the downstream flanking region (DFR), were amplified from MW0567 gDNA. Mutagenesis was carried out using site-directed mutagenesis, with primers designed to amplify the $\mathrm{MbsJ}^{\mathrm{C} 25 \mathrm{~A}}$ mutation. The primers used are listed in Supplementary Table 4. The fragments were assembled using overlapping PCR and the insertion cassette was transformed into the intergenic space between PF0265 and PF0266 of MW0011. The transformants were grown as previously described $^{49}$ and the PCR-confirmed colonies were sequence verified using Sanger sequencing (Genewiz)

Purification of C-MBS, activity assays, and EPR methods. The three C-MBS subcomplexes (WT, MbsL ${ }^{\mathrm{C} 85 \mathrm{~A} / \mathrm{C} 385 \mathrm{~A}}, \mathrm{MbsJ}^{\mathrm{C} 25 \mathrm{~A}}$ ) were purified anaerobically as described previously ${ }^{19}$. Frozen cells were lysed in $25 \mathrm{mM}$ sodium phosphate, pH 7.5, containing $1 \mathrm{mM}$ DTT and $50 \mu \mathrm{g} / \mu \mathrm{L}$ DNase I ( $5 \mathrm{~mL}$ per gram of frozen cells). After stirring for one hour, the cell-free extract was centrifuged at $100,000 \times \mathrm{g}$ for one hour. The supernatant was applied directly to a $5 \mathrm{~mL}$ His-Trap FF Ni-NTA column (GE Healthcare) by diluting it 10-fold with $25 \mathrm{mM}$ sodium phosphate, $\mathrm{pH} 7.5$ containing $300 \mathrm{mM} \mathrm{NaCl}$ and $1 \mathrm{mM}$ DTT (Buffer C). The column was equilibrated with Buffer $\mathrm{C}$ before loading the sample. The column was washed with 5 column volumes of Buffer $\mathrm{C}$ and the bound protein was eluted with a 20 -column volume gradient from 0 to $100 \%$ Buffer D (Buffer C containing $500 \mathrm{mM}$ imidazole).

All activity assays were carried out at $80^{\circ} \mathrm{C}$ using anaerobic sealed cuvettes as described previously ${ }^{19}$. The dimethyl trisulfide (DMTS) reduction assay used a $2 \mathrm{~mL}$ reaction mixture containing $100 \mathrm{mM} 3$-(N-morpholino) propanesulfonic acid (MOPS), $\mathrm{pH} 7.5$, and $150 \mathrm{mM} \mathrm{NaCl}$. After pre-heating to $80^{\circ} \mathrm{C}, 1 \mathrm{mM}$ methyl viologen reduced by titanium citrate and $2 \mathrm{mM}$ DMTS were added and the reaction was initiated by the addition of enzyme. For inactivation by cluster conversion, the enzyme $(2 \mathrm{mg} / \mathrm{mL}$ in $25 \mathrm{mM}$ sodium phosphate, $\mathrm{pH} 7.5)$ was incubated anaerobically with potassium ferricyanide $(1 \mathrm{mM})$ for $\sim 90 \mathrm{~h}$ at room temperature. The sample was then buffer exchanged to remove excess ferricyanide before measuring activity. To activate the enzyme, a mixture of sodium dithionite $(1 \mathrm{mM})$ and $\mathrm{FeCl}_{3}(1 \mathrm{mM})$ were added and after $\sim 90 \mathrm{~h}$ at room temperature, the sample was buffer exchanged to remove the excess reagent. Enzyme activity was measured by the reduction of DMTS by monitoring the oxidation of methyl viologen at $600 \mathrm{~nm}\left(\varepsilon=8.25 \mathrm{mM}^{-1} \mathrm{~cm}^{-1}\right)$ as previously described ${ }^{19}$. One unit of activity is defined as $1 \mu \mathrm{mol}$ of DMTS reduced per min.

All samples for spectroscopic studies were prepared under strictly anaerobic conditions. X-band $(\sim 9.6 \mathrm{GHz})$ EPR spectra were recorded using a Bruker EMXplus CW EPR spectrometer controlled with a Bruker PremiumX Ultra low noise microwave bridge, equipped with a cryogen-free ColdEdge stinger system and a Lakeshore temperature controller.

Cryo-EM analysis and data acquisition. MBS was purified using detergent DDM and was concentrated to $\sim 5 \mathrm{mg} / \mathrm{ml}$. Cryo-EM grids were prepared under the aerobic conditions and the processes took 20-30 $\mathrm{min}$ (MBS in air has a half-life of $19 \mathrm{~h}^{19}$ ). In brief, three-microliter aliquots of the sample were applied to glow-discharged Quantfoil R 1.2/1.3 gold grids ( 300 mesh). The grids were blotted for $3 \mathrm{~s}$ at $10^{\circ} \mathrm{C}$ with $100 \%$ humidity and were flash-frozen in liquid ethane using an FEI Vitrobot Mark IV device. Cryo-EM data were recorded on a K2 camera positioned post a GIF quantum energy filter in a $300 \mathrm{kV}$ FEI Titan Krios electron microscopy. Two datasets were automatically collected with Serial EM 3.7beta and FEI EPU software package, respectively, containing 8352 and 6355 movies respectively. Micrographs were recorded in counting mode at a nominal magnification of $130,000 \times$ with a pixel size of $1.03 \AA$ on sample. Defocus values varied from -1.1 to $-3 \mu \mathrm{m}$. The dose rate was 10 electrons per pixel per second. A total exposure of $6 \mathrm{~s}$ was dose-fractionated into 30 sub-frames, resulting in a total accumulated dose of 52 electrons per $\AA^{2}$.

Image processing. The two datasets collected on Titan Krios were processed using a similar strategy. Dose-fractionated movies with a physical pixel size of $1.03 \AA$ were motion-corrected and dose-weighted with MotionCor2 $1.1^{51,52}$. The contrast transfer function (CTF) parameter for individual micrograph was estimated by CTFFIND4.1.10 ${ }^{53}$. Further processing steps were carried out using RELION-3.0 ${ }^{54}$ For each dataset, a small set of particles were manually picked and were subjected to $2 \mathrm{D}$ classification. This generated templates for further reference-based automatic particle picking. Particle sorting and reference-free 2D classification were applied to the auto-picked particles for the removal of bad particles, resulting in 676,320 and 667,428 particles from the two respective datasets. The further $3 \mathrm{D}$ classification was performed on these cleaned-up particles using an ab initio map generated by cryoSPARC v2 as the initial mode ${ }^{55}$. For each dataset, three of four 3D classes were combined to get 467,320 and 419,868 good particles, respectively. Those were subjected to another round of 3D classification. One 3D class from each dataset with the best density features were combined. The combined dataset $(203,673$ particles) were used for 3D auto-refinement following CTF refinement, resulting in a map with an overall resolution of $4.0 \AA$ when twofold symmetry was applied. We note that most regions, particularly in the peripheral arm and the proximal membrane module, are of substantially better resolution in the range of 3.0-3.5. Some strategies including applying $\mathrm{C} 1$ symmetry, masking out detergent micelle or expanding the symmetry failed to improve the map. We therefore reasoned that the low overall resolution ( $4 \AA$ ) may be due to the partially flexible distal membrane region. The resolution of the reconstructed map was estimated based on the goldstandard Fourier shell correlation 0.143 criterion $^{56}$. The final map was corrected for the modulation transfer function of the detector and sharpened by applying a negative B-factor, estimated by the post-processing procedure in RELION-3.0. The estimate of Local resolution distribution was calculated using ResMap 1.1.5

Model building and refinement. The initial models of the distal module (MbsA-C, MbsE TMH3-6) and membrane-anchored hydrogenase module (MbsJ-N) were generated with the SWISS-MODEL server ${ }^{58}$ using the structure of $P$. furiosus $\mathrm{MBH}$ complex (PDB ID 6CFW) as the template. The initial model of the proximal module (MbsD, MbsE TMH3-6, MbsG, MbsH, and $\mathrm{MbsH}^{\prime}$ ) were generated using the structure of T. thermophilus complex I as a template (PDB ID 4HEA). These models were individually rigid-body docked into the $3 \mathrm{D}$ density map using the Fit-in-map function in Chimera $1.8^{59}$. These fitted models were improved by manual adjustments and rebuilding in Coot $0.8 .9^{60}$. The rebuilding process was aided by the good density features of a helices and many bulky residues such as Phe, Trp, Tyr, and Arg. The clusters within the peripheral arm were modeled based on their electron densities and also the homology of cluster-coordinating subunits with complex I and $\mathrm{MBH}$. The real-space refinement of the MBS complex model against the cryoEM map was performed using the phenix.real_space_refine in PHENIX $1.15^{61}$. MolProbity 4.1 (Duke University) was used to assess the final model ${ }^{62}$. Chimera and PyMOL 1.8 (Schrödinger, LLC.) were used to prepare the Figures. Statistics of the 3D reconstruction and model refinement were provided in Supplementary Table 1 Since all the subunits of Mrp antiporters have their counterparts in MBS (Supplementary Table 2), the structure of B. subtilis Mrp antiporter was modeled based on the MBS structure using the SWISS-MODEL server ${ }^{58}$

Reporting summary. Further information on research design is available in the Nature Research Reporting Summary linked to this article.

\section{Data availability}

The accession number for the atomic coordinates reported in this paper is PDB ID 6U8Y. The accession number for the EM density map reported in this paper is EMD-20692. Source data are provided with this paper. Other data are available from the corresponding authors upon reasonable request. Source data are provided with this paper.

Received: 3 August 2020; Accepted: 19 October 2020; Published online: 23 November 2020

\section{References}

1. Baradaran, R., Berrisford, J. M., Minhas, G. S. \& Sazanov, L. A. Crystal structure of the entire respiratory complex I. Nature 494, 443-448 (2013).

2. Zickermann, V. et al. Structural biology. Mechanistic insight from the crystal structure of mitochondrial complex I. Science 347, 44-49 (2015).

3. Zhu, J., Vinothkumar, K. R. \& Hirst, J. Structure of mammalian respiratory complex I. Nature 536, 354-358 (2016).

4. Fiedorczuk, K. et al. Atomic structure of the entire mammalian mitochondrial complex I. Nature 538, 406-410 (2016)

5. Guo, R., Zong, S., Wu, M., Gu, J. \& Yang, M. Architecture of human mitochondrial respiratory megacomplex I2III2IV2. Cell 170, 1247-1257.e1212 (2017).

6. Yu, H. et al. Structure of an ancient respiratory system. Cell 173, 1636-1649. e1616 (2018).

7. Sazanov, L. A. A giant molecular proton pump: structure and mechanism of respiratory complex I. Nat. Rev. Mol. Cell Biol. 16, 375-388 (2015).

8. Hirst, J. Mitochondrial complex I. Annu. Rev. Biochem. 82, 551-575 (2013).

9. Schut, G. J. et al. The role of geochemistry and energetics in the evolution of modern respiratory complexes from a proton-reducing ancestor. Biochim Biophys. Acta 1857, 958-970 (2016).

10. Letts, J. A. \& Sazanov, L. A. Clarifying the supercomplex: the higher-order organization of the mitochondrial electron transport chain. Nat. Struct. Mol. Biol. 24, 800-808 (2017).

11. Agip, A. A., Blaza, J. N., Fedor, J. G. \& Hirst, J. Mammalian respiratory Complex I through the lens of cryo-EM. Ann. Rev. Biophys. 48, 165-184 (2019).

12. Sapra, R., Bagramyan, K. \& Adams, M. W. A simple energy-conserving system: proton reduction coupled to proton translocation. Proc. Natl Acad. Sci. USA 100, 7545-7550 (2003).

13. Mayer, F. \& Muller, V. Adaptations of anaerobic archaea to life under extreme energy limitation. FEMS Microbiol. Rev. 38, 449-472 (2014). 
14. Kim, Y. J. et al. Formate-driven growth coupled with $\mathrm{H}(2)$ production. Nature 467, 352-355 (2010).

15. Lim, J. K., Mayer, F., Kang, S. G. \& Muller, V. Energy conservation by oxidation of formate to carbon dioxide and hydrogen via a sodium ion current in a hyperthermophilic archaeon. Proc. Natl Acad. Sci. USA 111, 11497-11502 (2014).

16. Laughlin, T. G., Bayne, A. N., Trempe, J. F., Savage, D. F. \& Davies, K. M. Structure of the complex I-like molecule NDH of oxygenic photosynthesis. Nature 566, 411-414 (2019).

17. Nisbet, E. G. \& Sleep, N. H. The habitat and nature of early life. Nature 409, 1083-1091 (2001).

18. McTernan, P. M. et al. Intact functional fourteen-subunit respiratory membrane-bound [NiFe]-hydrogenase complex of the hyperthermophilic archaeon Pyrococcus furiosus. J. Biol. Chem. 289, 19364-19372 (2014).

19. Wu, C. H., Schut, G. J., Poole, F. L. 2nd, Haja, D. K. \& Adams, M. W. W. Characterization of membrane-bound sulfane reductase: a missing link in the evolution of modern day respiratory complexes. J. Biol. Chem. 293, 16687-16696 (2018).

20. Schut, G. J., Bridger, S. L. \& Adams, M. W. Insights into the metabolism of elemental sulfur by the hyperthermophilic archaeon Pyrococcus furiosus: characterization of a coenzyme A- dependent $\mathrm{NAD}(\mathrm{P}) \mathrm{H}$ sulfur oxidoreductase. J. Bacteriol. 189, 4431-4441 (2007).

21. Conover, R. C. et al. Spectroscopic characterization of the novel iron-sulfur cluster in Pyrococcus furiosus ferredoxin. J. Biol. Chem. 265, 8533-8541 (1990).

22. Schicho, R. N., Ma, K., Adams, M. W. \& Kelly, R. M. Bioenergetics of sulfur reduction in the hyperthermophilic archaeon Pyrococcus furiosus. J. Bacteriol. 175, 1823-1830 (1993).

23. Enemark, J. H. \& Cosper, M. M. Molybdenum enzymes and sulfur metabolism. Met. Ions Biol. Syst. 39, 621-654 (2002).

24. Jormakka, M. et al. Molecular mechanism of energy conservation in polysulfide respiration. Nat. Struct. Mol. Biol. 15, 730-737 (2008).

25. Efremov, R. G. \& Sazanov, L. A. The coupling mechanism of respiratory complex I - a structural and evolutionary perspective. Biochim Biophys. Acta 1785-1795, 2012 (1817).

26. Hedderich, R. Energy-converting [NiFe] hydrogenases from archaea and extremophiles: ancestors of complex I. J. Bioenerg. Biomembr. 36, 65-75 (2004).

27. Schut, G. J., Boyd, E. S., Peters, J. W. \& Adams, M. W. The modular respiratory complexes involved in hydrogen and sulfur metabolism by heterotrophic hyperthermophilic archaea and their evolutionary implications. FEMS Microbiol Rev. 37, 182-203 (2013).

28. Di Luca, A., Gamiz-Hernandez, A. P. \& Kaila, V. R. I. Symmetry-related proton transfer pathways in respiratory complex I. Proc. Natl Acad. Sci. USA 114, E6314-E6321 (2017).

29. Kaila, V. R. I. Long-range proton-coupled electron transfer in biological energy conversion: towards mechanistic understanding of respiratory complex I. J. R. Soc., Interface 15, 20170916 (2018).

30. Euro, L., Belevich, G., Verkhovsky, M. I., Wikstrom, M. \& Verkhovskaya, M. Conserved lysine residues of the membrane subunit NuoM are involved in energy conversion by the proton-pumping NADH:ubiquinone oxidoreductase (Complex I). Biochim Biophys. Acta 1777, 1166-1172 (2008).

31. Brandt, U. Adaptations of an ancient modular machine. Science 363, 230-231 (2019).

32. Agip, A. A. et al. Cryo-EM structures of complex I from mouse heart mitochondria in two biochemically defined states. Nat. Struct. Mol. Biol. 25, 548-556 (2018).

33. Efremov, R. G. \& Sazanov, L. A. Structure of the membrane domain of respiratory complex I. Nature 476, 414-420 (2011).

34. Morino, M. et al. Single site mutations in the hetero-oligomeric Mrp antiporter from alkaliphilic Bacillus pseudofirmus OF4 that affect $\mathrm{Na}+\mathrm{H}+$ antiport activity, sodium exclusion, individual Mrp protein levels, or Mrp complex formation. J. Biol. Chem. 285, 30942-30950 (2010).

35. Steiner, J. \& Sazanov, L. Structure and mechanism of the Mrp complex, an ancient cation/proton antiporter. Elife 9, e59407 (2020).

36. Page, C. C., Moser, C. C., Chen, X. \& Dutton, P. L. Natural engineering principles of electron tunnelling in biological oxidation-reduction. Nature 402, 47-52 (1999).

37. Volbeda, A. et al. Structure of the [NiFe] hydrogenase active site: evidence for biologically uncommon Fe ligands. J. Am. Chem. Soc. 118, 12989-12996 (1996).

38. Schuller, J. M. et al. Structural adaptations of photosynthetic complex I enable ferredoxin-dependent electron transfer. Science 363, 257-260 (2019).

39. Berrisford, J. M. \& Sazanov, L. A. Structural basis for the mechanism of respiratory complex I. J. Biol. Chem. 284, 29773-29783 (2009).

40. Fontecilla-Camps, J. C., Volbeda, A., Cavazza, C. \& Nicolet, Y. Structure/ function relationships of [NiFe]- and [FeFe]-hydrogenases. Chem. Rev. 107, 4273-4303 (2007).

41. Kashani-Poor, N., Zwicker, K., Kerscher, S. \& Brandt, U. A central functional role for the $49-\mathrm{kDa}$ subunit within the catalytic core of mitochondrial complex I. J. Biol. Chem. 276, 24082-24087 (2001).
42. Wagner, T., Koch, J., Ermler, U. \& Shima, S. Methanogenic heterodisulfide reductase (HdrABC-MvhAGD) uses two noncubane [4Fe-4S] clusters for reduction. Science 357, 699-703 (2017).

43. Watanabe, $\mathrm{S}$. et al. Crystal structures of [NiFe] hydrogenase maturation proteins HypC, HypD, and HypE: Insights into cyanation reaction by thiol redox signaling. Mol. Cell 27, 29-40 (2007).

44. Walters, E. M. et al. Role of histidine-86 in the catalytic mechanism of ferredoxin: thioredoxin reductase. Biochemistry 48, 1016-1024 (2009).

45. Torres-Bacete, J., Nakamaru-Ogiso, E., Matsuno-Yagi, A. \& Yagi, T. Characterization of the NuoM (ND4) subunit in Escherichia coli NDH-1: conserved charged residues essential for energy-coupled activities. J. Biol. Chem. 282, 36914-36922 (2007).

46. Dey, A. et al. Solvent tuning of electrochemical potentials in the active sites of HiPIP versus ferredoxin. Science 318, 1464-1468 (2007).

47. Galkin, A., Drose, S. \& Brandt, U. The proton pumping stoichiometry of purified mitochondrial complex I reconstituted into proteoliposomes. Biochim Biophys. Acta 1757, 1575-1581 (2006).

48. Cabrera-Orefice, A. et al. Locking loop movement in the ubiquinone pocket of complex I disengages the proton pumps. Nat. Commun. 9, 4500 (2018).

49. Lipscomb, G. L. et al. Natural competence in the hyperthermophilic archaeon Pyrococcus furiosus facilitates genetic manipulation: construction of markerless deletions of genes encoding the two cytoplasmic hydrogenases. Appl Environ. Microbiol. 77, 2232-2238 (2011).

50. Bryksin, A. V. \& Matsumura, I. Overlap extension PCR cloning: a simple and reliable way to create recombinant plasmids. Biotechniques 48, 463-465 (2010).

51. Grant, T. \& Grigorieff, N. Measuring the optimal exposure for single particle cryo-EM using a 2.6 A reconstruction of rotavirus VP6. Elife 4, e06980 (2015).

52. Zheng, S. Q. et al. MotionCor2: anisotropic correction of beam-induced motion for improved cryo-electron microscopy. Nat. Methods 14, 331-332 (2017).

53. Rohou, A. \& Grigorieff, N. CTFFIND4: Fast and accurate defocus estimation from electron micrographs. J. Struct. Biol. 192, 216-221 (2015).

54. Kimanius, D., Forsberg, B. O., Scheres, S. H. \& Lindahl, E. Accelerated cryoEM structure determination with parallelisation using GPUs in RELION-2. Elife 5, e18722 (2016).

55. Punjani, A., Rubinstein, J. L., Fleet, D. J. \& Brubaker, M. A. cryoSPARC: algorithms for rapid unsupervised cryo-EM structure determination. Nat. Methods 14, 290-296 (2017).

56. Rosenthal, P. B. \& Henderson, R. Optimal determination of particle orientation, absolute hand, and contrast loss in single-particle electron cryomicroscopy. J. Mol. Biol. 333, 721-745 (2003).

57. Kucukelbir, A., Sigworth, F. J. \& Tagare, H. D. Quantifying the local resolution of cryo-EM density maps. Nat. Methods 11, 63-65 (2014).

58. Arnold, K., Bordoli, L., Kopp, J. \& Schwede, T. The SWISS-MODEL workspace: a web-based environment for protein structure homology modelling. Bioinformatics 22, 195-201 (2006).

59. Pettersen, E. F. et al. UCSF Chimera-a visualization system for exploratory research and analysis. J. Comput. Chem. 25, 1605-1612 (2004).

60. Emsley, P., Lohkamp, B., Scott, W. G. \& Cowtan, K. Features and development of Coot. Acta Crystallogr. D. Biol. Crystallogr. 66, 486-501 (2010).

61. Adams, P. D. et al. PHENIX: a comprehensive Python-based system for macromolecular structure solution. Acta Crystallogr. D. Biol. Crystallogr. 66, 213-221 (2010)

62. Chen, V. B. et al. MolProbity: all-atom structure validation for macromolecular crystallography. Acta Crystallogr. D. Biol. Crystallogr. 66, $12-21(2010)$

\section{Acknowledgements}

Cryo-EM images were collected at the David Van Andel Advanced Cryo-Electron Microscopy Suite in the Van Andel Research Institute. We thank Soshawn A. Blair, Sydney Hallenbeck, and Michael K. Johnson of the University of Georgia for helpful discussions on the catalytic mechanism of MBS and for assistance in running EPR spectroscopy. The EPR spectrometer was funded by an instrument award (CHE1827968) from the National Science Foundation. This work was funded by grants from the Division of Chemical Sciences, Geosciences and Biosciences, Office of Basic Energy Sciences of the U.S. Department of Energy (DOE; DE-SC0020085 to H.L. and DE-FG0295ER20175 to M.W.W.A.)

\section{Author contributions}

H.L. and M.W.W.A. conceived and designed experiments. H.Y., D.K.H., G.J.S., C.-H.W., X.M., and G.Z. performed experiments. H.Y., D.K.H, G.J.S., M.W.W.A., and H.L. analyzed the data. H.Y., D.K.H., H.L., and M.W.W.A. wrote the manuscript

\section{Competing interests}

The authors declare no competing interests. 


\section{Additional information}

Supplementary information is available for this paper at https://doi.org/10.1038/s41467020-19697-7.

Correspondence and requests for materials should be addressed to H.L. or M.W.W.A.

Peer review information Nature Communications thanks Thorsten Friedrich, James A. Letts and the other, anonymous, reviewer(s) for their contribution to the peer review of this work.

Reprints and permission information is available at http://www.nature.com/reprints

Publisher's note Springer Nature remains neutral with regard to jurisdictional claims in published maps and institutional affiliations. (c) (i) Open Access This article is licensed under a Creative Commons Attribution 4.0 International License, which permits use, sharing, adaptation, distribution and reproduction in any medium or format, as long as you give appropriate credit to the original author(s) and the source, provide a link to the Creative Commons license, and indicate if changes were made. The images or other third party material in this article are included in the article's Creative Commons license, unless indicated otherwise in a credit line to the material. If material is not included in the article's Creative Commons license and your intended use is not permitted by statutory regulation or exceeds the permitted use, you will need to obtain permission directly from the copyright holder. To view a copy of this license, visit http://creativecommons.org/ licenses/by/4.0/.

(C) The Author(s) 2020, corrected publication 2021 